\title{
Untersuchungen über die Beeinflussung der planktischen Photosynthese durch anorganische Metallsalze im eutrophen Alpnachersee und der mesotrophen Horwer Bucht
}

\author{
Von ReNE GÄCHTER
}

Eidg. Anstalt für Wasserversorgung, Abwasserreinigung und Gewässerschutz (EAWAG), Dübendorf

Manuskript eingegangen am 20. Juli 1976

\author{
ABSTRACT \\ Heavy Metal Toxicity and Synergism to natural phytoplankton
}

The effects on phytoplankton photosynthesis of inorganic metal salts $\mathrm{HgCl}_{2}, \mathrm{CuSO}_{4}, \mathrm{Cd}\left(\mathrm{NO}_{3}\right)_{2}$, $\mathrm{ZnCl}_{2}$ and $\mathrm{Pb}\left(\mathrm{NO}_{3}\right)_{2}$ were studied over monthly intervals.

In experiments with individual metals, phytoplankton photosynthesis was not adversely affected if the concentration increase above background levels did not exceed $10^{-9}$ mole $\mathrm{Hg} / 1,5 \cdot 10^{-9}$ mole $\mathrm{Cu} / 1,2 \cdot 10^{-8}$ mole $\mathrm{Cd} / 1,5 \cdot 10^{-8}$ mole $\mathrm{Zn} / 1$ and $2 \cdot 10^{-7}$ mole $\mathrm{Pb} / 1$, respectively. However, when the concentration was increased by $5 \cdot 10^{-10}$ mole $\mathrm{Hg} / 1+5 \cdot 10^{-9}$ mole $\mathrm{Cu} / 1+5 \cdot 10^{-9} \mathrm{~mole} \mathrm{Cd} / \mathrm{l}+5 \cdot 10^{-8}$ mole $\mathrm{Zn} / 1+5 \cdot 10^{-8}$ mole $\mathrm{Pb} / 1$ photosynthesis was significantly reduced, due to a synergetic effect of the combined metals.

The influence of phytoplankton density, $\mathrm{pH}$-value, concentration of calcium, dissolved organic nitrogen and allochthonous debris on heavy metal toxicity was investigated. Changes in phytoplankton composition are believed to be the main reason for the seasonal variation in the toxic effects of heavy metals.

\section{Einleitung}

Die Quecksilber- und Cadmiumvergiftungsfälle in Japan [19, 33], die Quecksilbervergiftungen des Lake St. Clair [14] und verschiedener Gewässer in Schweden [11], welche Einschränkungen der Fischerei notwendig machten, zeigen, dass zu hohe Metallbelastung die Nutzungsmöglichkeiten von Gewässern beeinträchtigen. Hohe Metallkonzentrationen in Gewässern gefährden aber nicht nur den Menschen und andere terrestrische Organismen, die in ihrer Nahrungsversorgung auf aquatischen Systemen basieren, sondern es wurde vielfach nachgewiesen, dass auch aquatische Organismen gegenüber Schwermetallen sehr empfindlich reagieren.

HyNes [18], WuHRMANN und EICHENBERGER [36] und WiNNER et al. [35] haben gezeigt, dass erhöhte Metallbelastungen zu qualitativen und quantitativen Veränderungen in der Zusammensetzung natürlicher aquatischer Biozoenosen führen. Sedimentuntersuchungen ergaben, dass in jüngster Zeit die Metallbelastung vieler 
Gewässer stark zugenommen hat [11]. Es kann nicht ausgeschlossen werden, dass die erhöhten Metallkonzentrationen in diesen Gewässern ihre Biozoenosen latent beeinflussen. Qualitative und quantitative Veränderungen werden mangels Vergleichsmöglichkeiten oft nicht erkannt oder können wegen der gleichzeitigen Veränderung anderer Parameter (z. B. Konzentration an organischen Verbindungen oder anorganischen Nährsalzen) nicht eindeutig mit der erhöhten Konzentration eines oder mehrerer Schwermetalle begründet werden.

Um ökologisch begründete Gewässerschutzmassnahmen planen zu können, ist es nötig, die maximal tolerierbaren Metallkonzentrationen zu kennen, die in einem gegebenen Gewässertyp nicht nur ein Überleben aller im unbelasteten Gewässer auftretenden Organismen ermöglichen, sondern auch eine ungestörte Fortpflanzung und ein natürliches Gedeihen aller ihrer Entwicklungsstadien erlauben.

Untersuchungen an Fischen und Fischnährtieren haben ergeben, dass die toxische Wirkung von Metallen von verschiedensten Faktoren beeinflusst wird: zum Beispiel von der Art, dem Alter und dem physiologischen Zustand der Versuchstiere, der Konzentration anderer Kationen und Anionen im allgemeinen, der Wasserhärte, der Alkalinität, der Salinität im speziellen, aber auch vom $\mathrm{pH}$, der Temperatur, der Sauerstoffkonzentration und der Expositionszeit. Algen reagieren auf erhöhte Metallkonzentrationen um so empfindlicher, je niedriger die Individuendichte [28-30] und je niedriger der Gehalt des Nährmediums an komplexierenden Verbindungen ist [3, 9, 10, 21, 25, 28-30].

Bis heute wurden wenige Versuche unternommen, diese qualitativ gut dokumentierten Zusammenhänge zu quantifizieren [z. B. 7, 23, 26]. Dies führt dazu, dass trotz der Vielzahl von vorhandenen Einzelinformationen die zentrale Frage, wie stark ein bestimmtes Gewässer mit Metallen belastet werden dürfe, ohne die gesamte Biozönose oder einzelne ihrer Vertreter zu schädigen, nicht schlüssig beantwortet werden kann.

Es war daher das Ziel dieser Arbeit, am Beispiel von zwei Alpenrandseen die Wirkung von Metallsalzen auf die planktische Photosynthese zu studieren und zu überprüfen, ob die gesetzlich festgelegten Toleranzkonzentrationen [34] der generellen Forderung, dass keine Substanz in toxisch wirksamer Konzentration auftreten dürfe, genügen.

Tabelle 1. Toxizitätssequenz einiger Metalle für verschiedene Organismen.

Table 1. Order of heavy metal toxicity to various organisms.

\begin{tabular}{lll}
$\begin{array}{l}\text { Organismus } \\
\text { Organisms }\end{array}$ & $\begin{array}{l}\text { Toxizitätssequenz } \\
\text { Order of toxicity }\end{array}$ \\
\hline Aal & $\mathrm{Hg}>\mathrm{Cu}=\mathrm{Zn}=\mathrm{Cd}>\mathrm{Sn}=\mathrm{Al}=\mathrm{Ni}$ \\
Eel & $\mathrm{Hg}=\mathrm{Cu}>\mathrm{Au}=\mathrm{Pd}>\mathrm{Th}>\mathrm{Pt}>\mathrm{Cd}$ \\
Zahnkarpfen & $\mathrm{Hg} \geqslant \mathrm{Cd}>\mathrm{Cu}>\mathrm{Zn}>\mathrm{Pb}>\mathrm{Ni}$ \\
Cyprinodont fish & $\mathrm{Hg} \geqslant \mathrm{Cu} \geqslant \mathrm{Ni}>\mathrm{Cd} \geqslant \mathrm{Zn}$ \\
Daphnia magna & $\mathrm{Ag}>\mathrm{Cd}>\mathrm{Ni}>\mathrm{Se}>\mathrm{Cu}>\mathrm{Ba}>\mathrm{Pb}$ \\
Microregma sp. & $\mathrm{Hg}>\mathrm{Cd}>\mathrm{Pb}>\mathrm{Cu}>\mathrm{Ni}=\mathrm{Zn}$ \\
Scenedesmus & $\mathrm{Ag}>\mathrm{Cd}>\mathrm{Cu}>\mathrm{Hg}>\mathrm{Se}>\mathrm{Ni}>\mathrm{Co}>\mathrm{Ba}>\mathrm{Pb}$ \\
&
\end{tabular}


Aufgrund der relativen Toxizität einzelner Metalle gegenüber aquatischen Organismen (siehe Tab.1) wurden als Testmetalle Quecksilber, Kupfer, Zink und Cadmium ausgewählt. Blei wurde mit in die Reihe aufgenommen, weil dieses Metall als Treibstoffadditiv mit den Automobilabgasen in grossen Mengen in die Umwelt gelangt.

Als Untersuchungsobjekte wurden die mesotrophe Horwer Bucht und der eutrophe Alpnachersee ausgewählt, weil sie sich durch niedrige Metallkonzentrationen auszeichnen [1] und weil sie bezüglich Härte, Alkalinität und Trophiegrad typisch sind für Seen im Alpennordrandgebiet. Tabelle 2 gibt eine grobe Charakterisierung der beiden Seen. Detailliertere Informationen finden sich bei Gächter [12], Stadelmann [27], KRUMmenacher [20] und Baccini [1].

Tabelle 2. Charakterisierung des Epilimnions der untersuchten Seen.

Table 2. Primary production and epilimnion-chemistry of the two lakes.

\begin{tabular}{|c|c|c|}
\hline & $\begin{array}{l}\text { Alpnachersee } \\
\text { Lake of Alpnach }\end{array}$ & $\begin{array}{l}\text { Horwer Bucht } \\
\text { Lake of Lucerne }\end{array}$ \\
\hline Chlorophyll $\left(\mathrm{mg} / \mathrm{m}^{3}\right)$ & $\begin{array}{ll}1 & -32\end{array}$ & $2-14$ \\
\hline 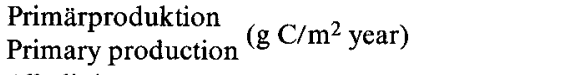 & 310 & 170 \\
\hline Alkalinität $_{\text {(mval/l) }}$ & $2.3-2.8$ & $1.6-1.9$ \\
\hline $\begin{array}{l}\text { Ca-Konzentration (mval/l) } \\
\text { Ca concentration }\end{array}$ & $2.8-3.4$ & $1.7-2.2$ \\
\hline $\mathrm{pH}$ & $\begin{array}{ll}7.6 & -8.7\end{array}$ & $7.6-8.7$ \\
\hline Gelöster organischer Kohlenstoff DOC mg C/l & $1.6-2.3$ & $0.7-1.9$ \\
\hline $\begin{array}{l}\text { Gelöster organischer Stickstoff DON } \mu \mathrm{g} \mathrm{N} / 1 \\
\text { L }\end{array}$ & $100-280$ & $60-220$ \\
\hline $\begin{array}{l}\text { Kupfer } \\
\text { Copper }\end{array}\left(10^{-8} \mathrm{Mol} / \mathrm{l}\right)$ & $0.9-1.8$ & $0.7-1.9$ \\
\hline Cadmium $\left(10^{-8} \mathrm{Mol} / \mathrm{l}\right)$ & $0.01-0.04$ & $0.01-0.04$ \\
\hline Blei $\left(10^{-8} \mathrm{Mol} / \mathrm{l}\right)$ & $<0.2-0.6$ & $0.2-0.6$ \\
\hline Zink $\left(10^{-8} \mathrm{Mol} / 1\right)$ & $2.0-6.0$ & $1.6-5.0$ \\
\hline
\end{tabular}

\section{Methodik}

Die Reaktion des Phytoplanktons auf erhöhte Metallkonzentrationen wurde in monatlichen Intervallen untersucht. Zu diesem Zweck wurde eine aus etwa $20 \mathrm{~cm}$ Tiefe entnommene Probe in 40 Teilproben unterteilt. Durch Zugabe von $\mathrm{HgCl}_{2}$, $\mathrm{CuSO}_{4}, \mathrm{Cd}\left(\mathrm{NO}_{3}\right)_{2}, \mathrm{ZnCl}_{2}$ und $\mathrm{Pb}\left(\mathrm{NO}_{3}\right)_{2}$ wurde in ihnen die Metallkonzentration um den in Tabelle 3 angegebenen Betrag erhöht. Wurde die Konzentration aller fünf Metalle gleichzeitig angehoben, so war die Konzentrationserhöhung eines jeden Metalls 10mal niedriger als bei den Versuchen mit den reinen Lösungen. Anschliessend wurden die Proben mit $\mathrm{C}_{14}$-Bikarbonatlösung versetzt und während 24 Stunden im Rotationslichtthermostaten bei der im See gemessenen aktuellen Temperatur exponiert. Danach wurden $25 \mathrm{ml}$ jeder Probe durch ein SartoriusMembranfilter $(0,3 \mu \mathrm{m})$ filtriert, die Filter in ein Zählschälchen geklebt und nach 
dem Trocknen während 20 Minuten über rauchender Salzsäure exponiert, um anorganisches adsorbiertes $\mathrm{C}_{14}$ auszutreiben. Die Messung der $\mathrm{C}_{14}$-Aktivität erfolgte im Methandurchflusszähler FH407 der Firma Frieseke und Höpfner.

Tabelle 3. Erhöhung der Metallkonzentration in den Teilproben.

Table 3. Increase of heavy metal concentration in the subsamples.

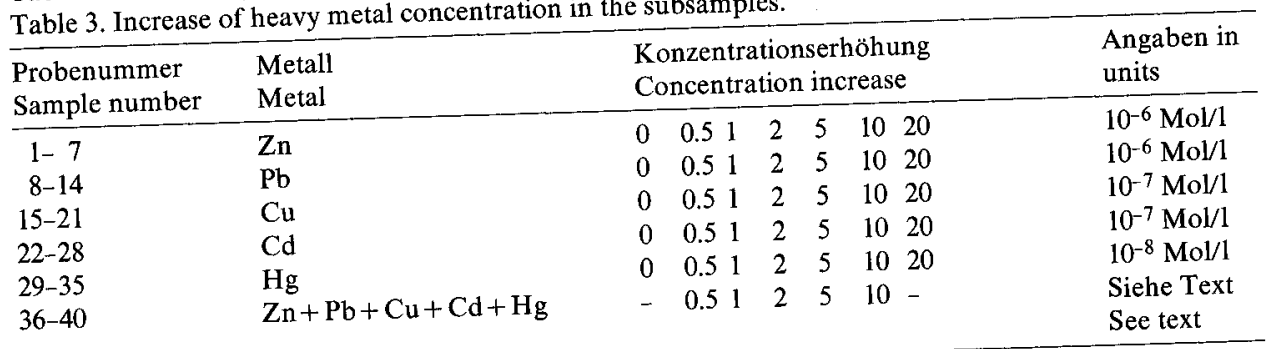

\section{Resultate}

Die Resultate von etwa 800 Einzelmessungen wurden in Abb. 1 graphisch zusammengefasst. Es zeigt sich dabei,

- dass eine bestimmte Konzentrationserhöhung ein und desselben Metalls je nach Jahreszeit die Photosyntheseleistung des Planktons unterschiedlich stark hemmte,

- dass die Metalle in den beiden Seen teilweise unterschiedlich toxisch wirkten,

- dass eine gleichzeitige Erhöhung der Konzentration mehrerer Metalle zu einer additiven oder sogar zu einer synergistischen Wirkungssteigerung der einzelnen Metalle führte,

- dass sich die verschiedenen Metalle bezüglich ihrer toxischen Wirkung zum Teil deutlich unterscheiden.

Cadmium und Zink hemmten die planktische Photosynthese in beiden Seen im Sommer (Mai bis September) am stärksten. Für Kupfer und Quecksilber ergaben sich mehrere synchrone Empfindlichkeitsmaxima im Verlaufe des Jahres, nämlich zwei in der Horwer Bucht (Februar/März und Juni) und vier im Alpnachersee (Februar/März, Juni, September und November/Dezember). Das Blei zeigte dagegen, was die saisonale Veränderung der toxischen Wirkung gegenüber dem Phytoplankton anbelangt, in beiden Seen keinerlei Übereinstimmung mit einem der andern Metalle. Das Phytoplankton beider Seen zeigte gegenüber Blei dann die grösste Resistenz, wenn es gegenüber allen andern Metallen maximal empfindlich reagierte, nämlich im Juni/Juli im Alpnachersee und im Juni in der Horwer Bucht. Umgekehrt hemmte eine Erhöhung der Bleikonzentration in beiden Seen im April die Photosyntheseleistung des Planktons am stärksten, zu einem Zeitpunkt also, wo die Auswirkungen aller anderen Metalle minimal waren.

In der Regel reagierte das Plankton des Alpnachersees weniger empfindlich auf Konzentrationserhöhungen von Cadmium, Zink und Blei als dasjenige der Horwer Bucht. Eine Konzentrationserhöhung von $5 \cdot 10^{-7} \mathrm{Mol}$ Cadmium hemmte die Photosyntheseleistung im Jahresmittel im Alpnachersee um 40\%, in der Horwer 

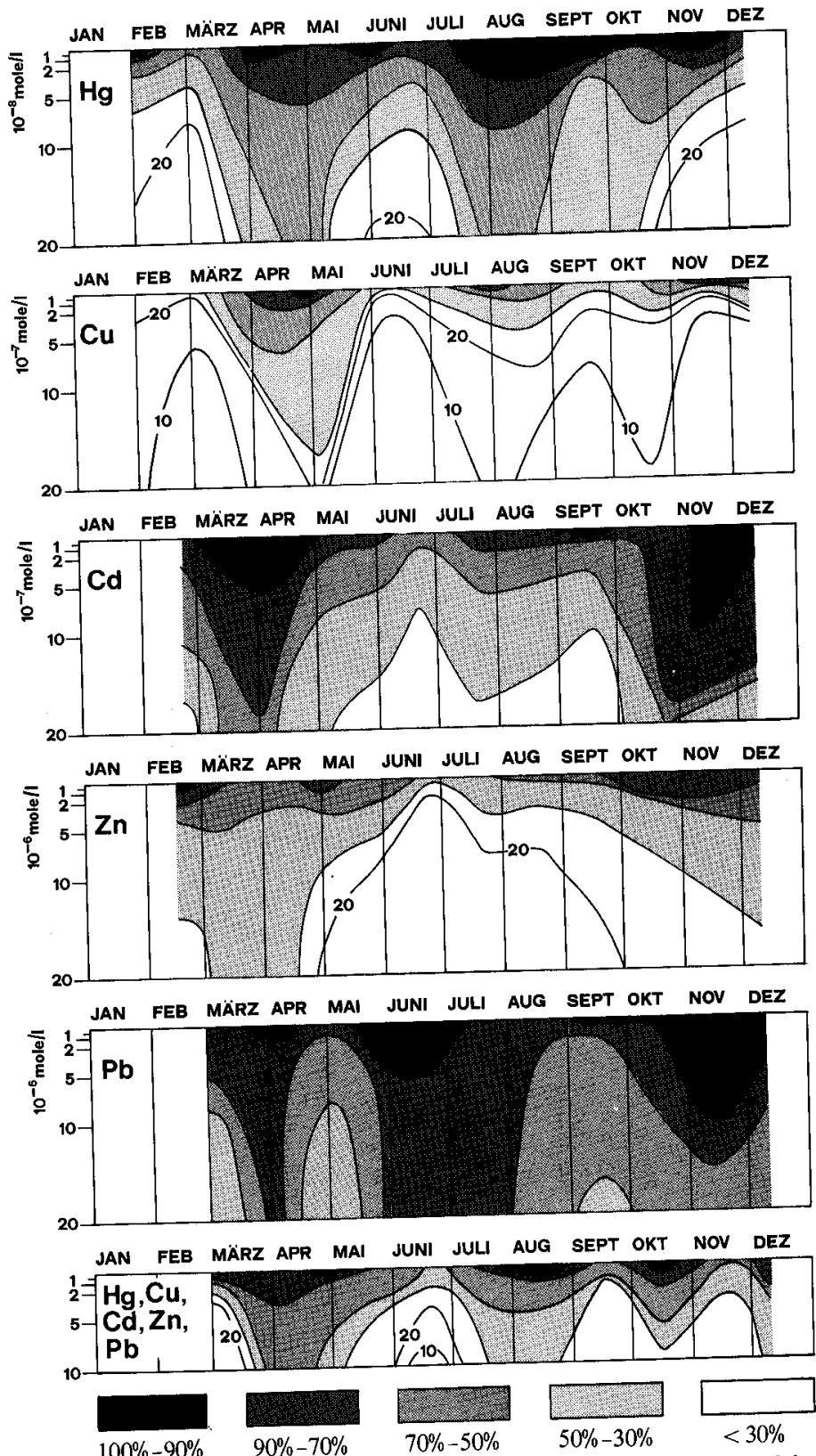

Abb.1a. Relative Photosyntheseraten im Alpnachersee in Abhängigkeit von der Jahreszeit und der

Konzentrationserhöhung einzelner Metalle (Kontrolle $=100 \%$ ).

Fig. 1a. Seasonal variation of relative photosynthesis (control $=100 \%$ ) in Lake of Alpnach as a function of increased heavy metal concentration.

The different shadows in Figures $1 \mathrm{a}$ and $1 \mathrm{~b}$ indicate (from black to light)

black 100 to $90 \%$ of control; 90 to $70 \%$ of control; 70 to $50 \%$ of control; 50 to $30 \%$ of control white less than $30 \%$ of control 

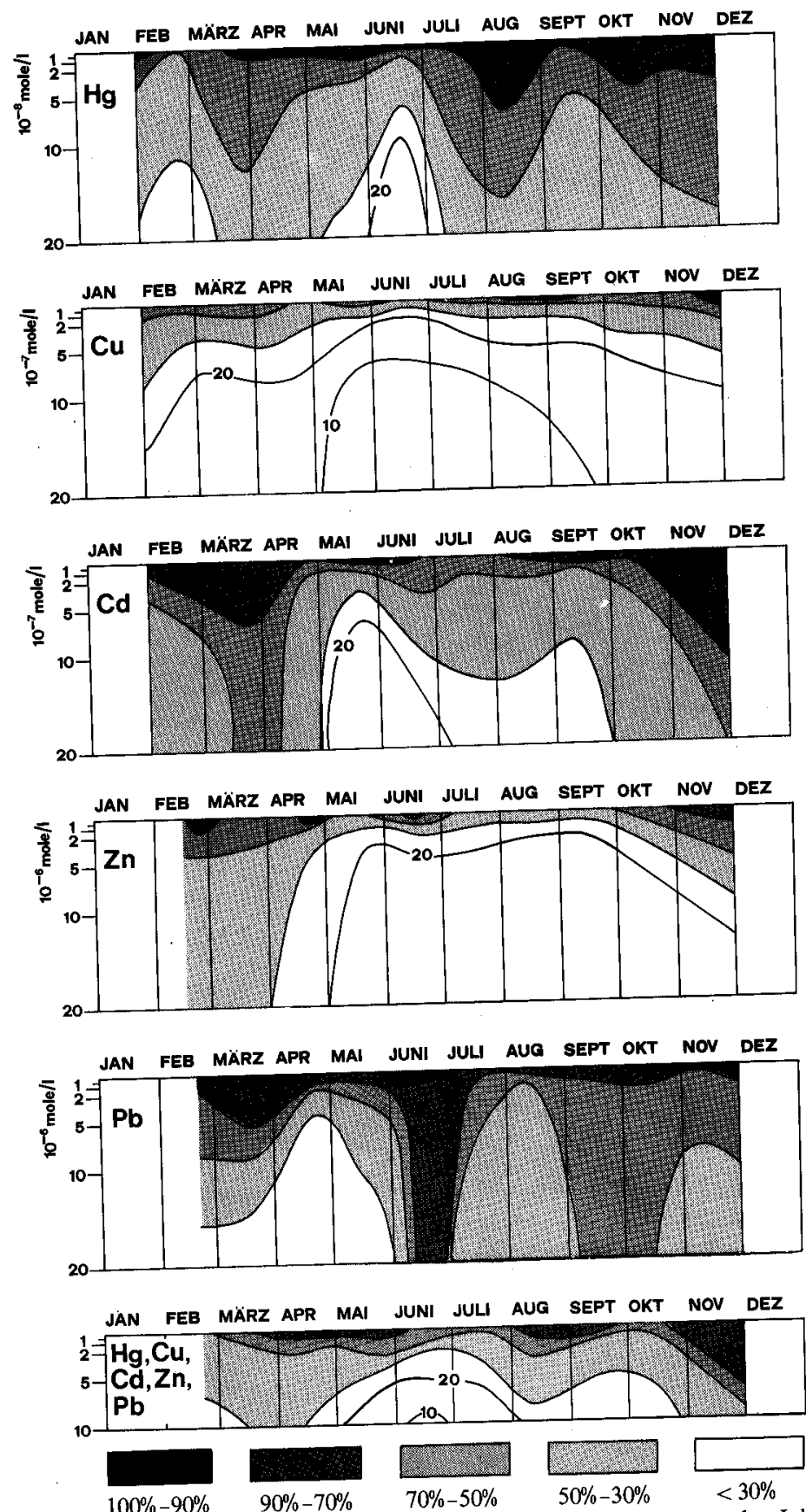

Abb.1b. Relative Photosyntheseraten in der Horwer Bucht in Abhängigkeit von der Jahreszeit und der

Konzentrationserhöhung einzelner Metalle (Kontrolle $=100 \%)$.

Fig. 1b. Seasonal variation of relative photosynthesis (control $=100 \%$ ) in Lake Lucerne as a function of increased heavy metal concentration. 
Bucht um 50\%. 5 $10^{-6}$ Mol Blei reduzierte die mittlere Photosyntheseleistung im Alpnachersee um etwa 20\%, in der Horwer Bucht dagegen um etwa $45 \%$. Eine Konzentrationserhöhung um $2 \cdot 10^{-6} \mathrm{Mol} \mathrm{Zn} / 1$ hemmte die Photosynthese im Alpnachersee im Jahresmittel um 50\%, in der Horwer Bucht dagegen um mehr als $60 \%$.

Im Gegensatz dazu reagierte das Alpnacherseeplankton im Februar/März und im November/Dezember deutlich empfindlicher auf eine Erhöhung der Quecksilberund Kupferkonzentration als das Plankton der Horwer Bucht. Im Jahresmittel konnte zwischen den beiden Seen aber kein signifikanter Unterschied festgestellt werden. $2 \cdot 10^{-7} \mathrm{Mol}$ Quecksilber reduzierte die Photosyntheseleistung in beiden Seen um etwa $70 \%, 10^{-7} \mathrm{Mol} \mathrm{Cu} / 1$ um etwa $60 \%$.

Bei gleichzeitiger Konzentrationserhöhung von $\mathrm{Hg}, \mathrm{Cu}, \mathrm{Cd}, \mathrm{Zn}$ und $\mathrm{Pb}$ wurde die Primärproduktion dann am stärksten gehemmt, wenn das Phytoplankton gleichzeitig gegenüber einem oder mehreren Einzelmetallen empfindlich reagierte. Empfindlichkeitsmaxima wurden daher im Alpnachersee im März (hohe Empfindlichkeit gegenüber $\mathrm{Hg}, \mathrm{Cu}, \mathrm{Cd}, \mathrm{Zn}, \mathrm{Pb}$ ) von $\mathrm{Mai}$ bis Juli (hohe Empfindlichkeit gegenüber $\mathrm{Hg}, \mathrm{Cu}, \mathrm{Cd}, \mathrm{Zn}$ ) im September (hohe Empfindlichkeit gegenüber $\mathrm{Hg}$, $\mathrm{Cu}, \mathrm{Cd}, \mathrm{Zn}, \mathrm{Pb}$ ) und im November (hohe Empfindlichkeit gegenüber $\mathrm{Hg}, \mathrm{Cu}$ ) beobachtet. In der Horwer Bucht ergab sich dagegen nur ein Hauptmaximum im Juni (hohe Empfindlichkeit gegenüber $\mathrm{Hg}, \mathrm{Cu}, \mathrm{Cd}, \mathrm{Zn}$ ) und zwei Nebenmaxima im Februar (hohe Empfindlichkeit gegenüber $\mathrm{Hg}$ ) und September (hohe Empfindlichkeit gegenüber $\mathrm{Cu}, \mathrm{Cd}, \mathrm{Zn}$ ).

Im Jahresmittel hemmte eine Kombination von $2 \cdot 10^{-9} \mathrm{Mol} \mathrm{Hg} / 1,2 \cdot 10^{-8} \mathrm{Mol}$ $\mathrm{Cu} / \mathrm{l}, 2 \cdot 10^{-8} \mathrm{Mol} \mathrm{Cd} / 1,2 \cdot 10^{-7} \mathrm{Mol} \mathrm{Zn} / 1$ und $2 \cdot 10^{-7} \mathrm{Mol} \mathrm{Pb} / 1$ die Primärproduktion in beiden Seen um etwa $50 \%$.

$\mathrm{Da}$ in diesem Konzentrationsbereich kein Metall für sich allein die Photosynthese so stark hemmt, ist die beobachtete Hemmung sicher das Resultat einer additiven oder sogar synergistischen Wirkung mehrerer Metalle.
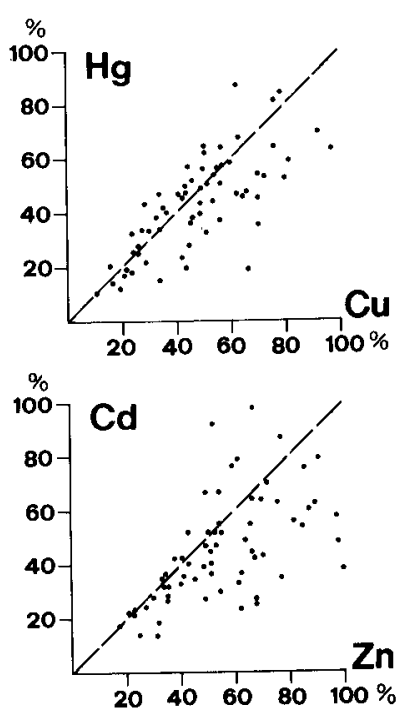
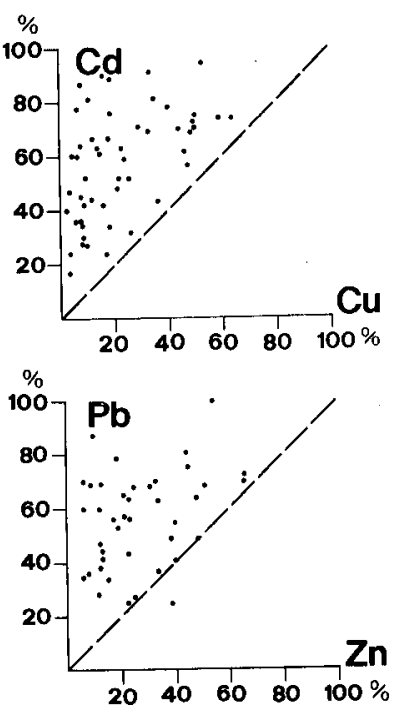

Abb.2. Relative Photosyntheseraten (Kontrolle $=100 \%$ ) bei äquimolarer Konzentrationserhöhung von $\mathrm{Hg}$ und $\mathrm{Cu}, \mathrm{Cd}$ und $\mathrm{Cu}$, $\mathrm{Cd}$ und $\mathrm{Zn}, \mathrm{Pb}$ und $\mathrm{Zn}$. Fig. 2. Relative photosynthesis (control, without additional metal $=100 \%$ ) at equimolar concentrations of $\mathrm{Hg}$ and $\mathrm{Cu}$, $\mathrm{Cd}$ and $\mathrm{Cu}, \mathrm{Cd}$ and $\mathrm{Zn}$, $\mathrm{Pb}$ and $\mathrm{Zn}$. 
Abb. 2 zeigt im paarweisen Vergleich die Auswirkungen von äquimolaren Konzentrationserhöhungen verschiedener Metallsalze auf die Photosyntheseleistung des Phytoplanktons. Es geht daraus hervor, dass Quecksilber und Kupfer bei äquimolarer Konzentrationserhöhung die Photosyntheseleistung etwa gleich stark hemmen, Kupfer jedoch toxischer wirkt als Cadmium, Cadmium hemmt die Primärproduktion stärker als Zink, und Zink wiederum wirkt bei äquimolarer Anwendung deutlich stärker hemmend als Blei. Bezüglich ihrer toxischen Wirkung ergibt sich für die untersuchten Metalle die folgende Sequenz

$$
\mathrm{Hg} \geqslant \mathrm{Cu}>\mathrm{Cd}>\mathrm{Zn}>\mathrm{Pb} \text {. }
$$

\section{Diskussion}

\subsection{Problematik bei der Festlegung von Toleranzkonzentrationen}

Algen nehmen mangels eines wirksamen Selektionsvermögens Metallionen weit über den physiologischen Bedarf hinaus proportional zur Konzentration in der Nährlösung auf $[6,13,22,24]$. Viele Schwermetalle zeigen eine grosse Affinität $\mathrm{zu}$ den besonders reaktionsfähigen Amino-, Imino- und Sulfydrylgruppen [5]. Es besteht daher die Möglichkeit, dass durch den Einbau von «falschen» Metallen in Enzyme deren Funktionsfähigkeit oder durch ihren Einbau in Zellmembranen deren Struktur und Permeabilität verändert werden. Aufgrund des Massenwirkungsgesetzes ist die Chance, dass derartige «Fehlkonstruktionen» und damit funktionelle Störungen auftreten, um so grösser, je mehr die Konzentration eines Metalls vom optimalen Konzentrationsbereich abweicht und je stabilere Verbindungen das Metallion mit den obgenannten funktionellen Gruppen eingeht.

Aufgrund der Reihenfolge der Elektronegativität $\mathrm{Hg}>\mathrm{Cu}>\mathrm{Pb}>\mathrm{Cd}>\mathrm{Zn}$, die auch mit der Stabilität der Chelatderivate der Metalle übereinstimmt, wäre bezüglich der Giftigkeit der verschiedenen Metalle die folgende Sequenz zu erwarten [5]:

$$
\mathrm{Hg}>\mathrm{Cu}>\mathrm{Pb}>\mathrm{Cd}>\mathrm{Zn} \text {. }
$$

Dies stimmt sehr gut mit der tatsächlich beobachteten Reihenfolge von

$$
\mathrm{HG} \geqslant \mathrm{Cu}>\mathrm{Cd}>\mathrm{Zn}>\mathrm{Pb}
$$

überein, wenn man berücksichtigt, dass ein Teil des zugegebenen Bleis rasch als trüber Niederschlag ausfiel und daher die physiologisch wirksamen Konzentrationserhöhungen sicher deutlich unter den nominell berechneten Werten lagen.

Die synergistische Wirkungssteigerung bei der gleichzeitigen Konzentrationserhöhung mehrerer Metalle (siehe S. 104ff.) und die Beobachtung, dass sich die Empfindlichkeit des Planktons gegenüber verschiedenen Metallen im Verlaufe des Jahres zum Teil asynchron veränderte, sprechen allerdings gegen die Annahme, dass die toxische Wirkung aller Metalle auf der gleichen Reaktion mit den gleichen Verbindungen beruhe und das Ausmass der Photosynthesehemmung nur von der Anzahl dieser «Fehlverbindungen» bestimmt werde. Aus der Beobachtung, dass die Empfindlichkeit des Planktons gegenüber Kupfer und Quecksilber und gegenüber Zink und Cadmium synchron variierte, kann vermutet werden, dass die Hemmung der Photosynthese durch Metalle auf mindestens 
zwei Wirkungsmechanismen beruht, wobei der eine vor allem durch Kupfer und Es wurde verschiedentlich beobachtet, dass ein Meeinflusst wird. leistung oder die Teilungsrate des Phytoplanktons zwar anfänglich hemmte, nach einiger Zeit sich aber weder die Photosyntheseleistung noch die Wachstumsrate von der Kontrolle unterschieden [28, 30, 31]. Als mögliche Mechanismen für die Entgiftung des Kulturmediums in Anwesenheit von Planktonorganismen wurden die Komplexierung der Metalle durch Exkretionsprodukte oder ihre Bindung an physiologisch inaktiven Stellen in oder an der Zelle diskutiert. Dass ein Metallstress in den ersten 24 Stunden keine, bei längerer Versuchsdauer aber eine erhebliche Reduktion von Photosynthese und Wachstum bewirkt hätte, wurde unseres Wissens aber nie beobachtet.

Mit Hilfe von Kurzzeitexperimenten von 24 Stunden Dauer lassen sich daher jene Metallkonzentrationen ermitteln, die mit hoher Wahrscheinlichkeit keinen negativen Einfluss auf das Phytoplankton ausüben. Ob Metallkonzentrationen, die während der gewählten Versuchsdauer eine spontane Reduktion der Photosynthese bewirken, das Phytoplankton anhaltend zu beeinflussen vermögen, muss dagegen im Langzeitexperiment untersucht werden. HORNE und GoldMAN [15] haben gezeigt, dass verschiedene Arten einer natürlichen Biozönose gegenüber Metallen unterschiedlich empfindlich reagieren. Es ist daher anzunehmen, dass Metallkonzentrationen, die eine spontane Reduktion der Photosyntheseleistung der Gesamtpopulation bewirken, mit grosser Wahrscheinlichkeit die qualitative Zusammensetzung des Planktons verändern.

Eine Reduktion der Primärproduktion um 20\% kann analytisch mit Sicherheit erfasst werden. Die Konzentrationserhöhungen $C_{24 \mathrm{~h}}^{20 \%}$, die diese Reduktion bewirken und daher einen eindeutig negativen Effekt auf das aktuell vorhandene Plankton ausüben, wurden in Tabelle 4 zusammengestellt. Konzentrationserhöhungen von $0,5 C_{24 \mathrm{~h}}^{20 \%}$ dürften die Photosyntheseleistung des Planktons um etwa $10 \%$ reduzieren.

Tabelle 4. Konzentrationserhöhungen $\left(C_{24 \mathrm{~h}}^{20 \%}\right)$, welche die planktische Photosynthese um $20 \%$ reduzieren $\left(10^{-8} \mathrm{Mol} / 1\right)$. A: Alpnachersee, H: Horwer Bucht.

Table 4. Increases of metal concentration which reduced primary production by $20 \%$. A: Lake of Alpnach, H: Lake of Lucerne. Figures indicate $10^{-8}$ mole $/ 1$.

\begin{tabular}{|c|c|c|c|c|c|c|c|c|c|c|}
\hline \multirow[t]{2}{*}{$\begin{array}{l}\text { Monat } \\
\text { Month }\end{array}$} & \multicolumn{2}{|l|}{$\begin{array}{l}\text { Zink } \\
\text { Zinc }\end{array}$} & \multicolumn{2}{|c|}{$\begin{array}{l}\text { Kupfer } \\
\text { Copper }\end{array}$} & \multicolumn{2}{|c|}{$\begin{array}{l}\text { Cadmium } \\
\text { Cadmium }\end{array}$} & \multicolumn{2}{|l|}{$\begin{array}{l}\text { Blei } \\
\text { Lead }\end{array}$} & \multicolumn{2}{|c|}{$\begin{array}{l}\text { Quecksilber } \\
\text { Mercury }\end{array}$} \\
\hline & $\mathrm{A}$ & $\mathrm{H}$ & A & $\mathrm{H}$ & A & $\mathrm{H}$ & A & $\mathbf{H}$ & A & $\mathrm{H}$ \\
\hline Feb. & 100 & 150 & 2 & 2 & 5 & 20 & 120 & 80 & 0.7 & 0.1 \\
\hline März & 40 & 40 & 1 & 3 & 20 & 8 & 200 & 100 & 0.2 & 0.5 \\
\hline April & 40 & 70 & 4 & 1 & 100 & 4 & 200 & 100 & 3 & 0.6 \\
\hline Juni & $\begin{array}{l}30 \\
14\end{array}$ & $\begin{array}{l}14 \\
40\end{array}$ & $\begin{array}{l}1 \\
2\end{array}$ & $\begin{array}{l}2 \\
3\end{array}$ & 4 & $\begin{array}{l}4 \\
4\end{array}$ & $\begin{array}{l}500 \\
700\end{array}$ & $\begin{array}{r}50 \\
100\end{array}$ & 2 & 1 \\
\hline Juli & 10 & 30 & 4 & $\begin{array}{l}3 \\
1\end{array}$ & 10 & $\begin{array}{l}4 \\
3\end{array}$ & 100 & $\begin{array}{r}100 \\
50\end{array}$ & 1 & $\begin{array}{l}0.4 \\
0.5\end{array}$ \\
\hline Aug. & 20 & 10 & 8 & $\begin{array}{l}1 \\
4\end{array}$ & 5 & $\begin{array}{l}3 \\
4\end{array}$ & 70 & $\begin{array}{l}50 \\
50\end{array}$ & $\begin{array}{l}6 \\
7\end{array}$ & $\begin{array}{l}0.5 \\
5\end{array}$ \\
\hline Sept. & 30 & 10 & 1 & $\begin{array}{l}4 \\
5\end{array}$ & 7 & $\begin{array}{l}4 \\
4\end{array}$ & 70 & $\begin{array}{r}50 \\
100\end{array}$ & $\begin{array}{l}7 \\
4\end{array}$ & $\begin{array}{l}5 \\
0.5\end{array}$ \\
\hline Okt. & 100 & 10 & 1 & $\begin{array}{l}5 \\
2\end{array}$ & $\begin{array}{l}10 \\
10\end{array}$ & 4 & 200 & 70 & 1 & $\begin{array}{l}0.5 \\
2\end{array}$ \\
\hline Nov. & 100 & 40 & 2 & $\begin{array}{l}2 \\
4\end{array}$ & $\begin{array}{r}10 \\
120\end{array}$ & 25 & 130 & 100 & 3 & \\
\hline Dez. & 120 & 150 & 2 & 12 & $\begin{array}{r}120 \\
30\end{array}$ & 50 & 80 & 20 & 1 & $\begin{array}{l}2 \\
3\end{array}$ \\
\hline
\end{tabular}



der angewandten Methodik nicht mit Sicherheit nachgewiesen werden kann, folgt aus den in Tabelle 4 zusammengestellten Resultaten, dass Konzentrationserhöhungen in der Grössenordnung von $0,1 \cdot 10^{-8} \mathrm{Mol} \mathrm{Hg} / 1,0,5 \cdot 10^{-8} \mathrm{Mol} \mathrm{Cu} / \mathrm{l}$, $2 \cdot 10^{-8} \mathrm{Mol} \mathrm{Cd} / 1,5 \cdot 10^{-8} \mathrm{Mol} \mathrm{Zn} / 1$ und $20 \cdot 10^{-8} \mathrm{Mol} \mathrm{Pb} / 1$ in beiden Seen während des ganzen Jahres keine messbare Reduktion der Photosyntheseleistung bewirken. Trotzdem können diese Konzentrationserhöhungen nicht als tolerierbar bezeichnet werden, weil die Versuche mit der kombinierten Metallzugabe ergaben, dass das, was für ein einzelnes Metall gilt, bei gleichzeitiger Konzentrationserhöhung mehrerer Metalle nicht mehr zutrifft. Konzentrationserhöhungen um nur 0,05 $10^{-8}$ $\mathrm{Mol} \mathrm{Hg} / 1+0,5 \cdot 10^{-8} \mathrm{Mol} \mathrm{Cu} / 1+0,5 \cdot 10^{-8} \mathrm{Mol} \mathrm{Cd} / 1+5 \cdot 10^{-8} \mathrm{Mol} \mathrm{Zn} / 1+5 \cdot 10^{-8}$ $\mathrm{Mol} \mathrm{Pb} / 1$ reduzierten die planktische Photosynthese während des Grossteils des Jahres in beiden Seen um deutlich mehr als $10 \%$ (siehe Tab. 5).

Tabelle 5. Prozentuale Reduktion der Photosyntheseraten bei gleichzeitiger Zugabe von $5 \cdot 10^{-10} \mathrm{Mol}$ $\mathrm{Hg} / 1,5 \cdot 10^{-9} \mathrm{Mol} \mathrm{Cu} / 1,5 \cdot 10^{-9} \mathrm{Mol} \mathrm{Cd} / 1,5 \cdot 10^{-8} \mathrm{Mol} \mathrm{Zn} / 1,5 \cdot 10^{-8} \mathrm{Mol} \mathrm{Pb} / 1$.

Table 5. Relative reduction of photosynthesis (control $100 \%$ ) when $\mathrm{Hg}, \mathrm{Cu}, \mathrm{Cd}, \mathrm{Zn}$ and $\mathrm{Pb}$ concentra$5 \cdot 10^{-9}$ mole $\mathrm{Cd} / 1,5 \cdot 10^{-8}$ mole $\mathrm{Zn} / 1,5 \cdot 10^{-8}$ mole $\mathrm{Pb} / \mathrm{l}$.

\begin{tabular}{|c|c|c|c|c|c|c|c|c|c|c|c|}
\hline & Feb. & März & April & Mai & Juni & Juli & Aug. & Sept. & Ukt. & Nuv. & \\
\hline $\begin{array}{l}\text { Horw } \\
\text { Lake of Lucerne }\end{array}$ & 49 & 27 & 6 & 16 & 43 & 30 & 11 & 19 & 37 & 16 & 0 \\
\hline $\begin{array}{l}\text { Alpnach } \\
\text { Lake of Alpnach }\end{array}$ & 27 & 25 & 9 & 33 & 59 & 13 & 9 & 26 & 7 & 33 & 21 \\
\hline
\end{tabular}

Diese Gegenüberstellung zeigt die Problematik, die sich bei der Festlegung von Toleranzkonzentrationen ergibt, wenn dabei nur auf Befunde von Untersuchungen mit einzelnen Metallen abgestellt wird. Um der additiven oder synergistischen Wirkung verschiedener Schadstoffe Rechnung zu tragen, sollte die Toleranzkonzentration für jedes Metall so festgelegt werden, dass sie deutlich unterhalb jener Konzentration liegt, die bei individueller Anwendung gerade keine unterhalb jener Konzentration liegt, die bei indigetzlich erlaubter Konzentrationsan-
messbare Schädigung mehr bewirkt. Ein geset
stieg in den Zuflüssen zum See um etwa $0,5 \cdot 10^{-8} \mathrm{Mol} \mathrm{Hg} / 1,15 \cdot 10^{-8} \mathrm{Mol} \mathrm{Cu} / \mathrm{l}$, $4 \cdot 10^{-8} \mathrm{Mol} \mathrm{Cd} / 1$ und $24 \cdot 10^{-8} \mathrm{Mol} \mathrm{Pb} / 1$ würde im See mit Sicherheit zu Metallkonzentrationen führen, die deutlich über den in Tabelle 5 angegebenen, nachgewiesenermassen toxischen Werten liegen. Es muss daher angenommen werden, dass Konzentrationserhöhungen einzelner oder mehrerer Metalle bereits unterhalb den in der Verordnung angegebenen Toleranzlimiten (Qualitätszielen) [34] nicht nur in Fliessgewässern [18, 35, 36], sondern auch in Seen erhebliche biozoenotische Veränderungen bewirken können.

Um zu überprüfen, ob sich die einzelnen Metalle bei gleichzeitiger Konzentrationserhöhung in ihrer Wirkung additiv oder synergistisch verhalten, wurden die Funktionen, welche die Photosynthesedepression des Planktons in Abhängigkeit von der Konzentrationserhöhung der Metalle beschreiben, normalisiert. Dabei wurde die Konzentrationserhöhung $C_{24 \mathrm{~h}}^{50 \%}$, welche die Photosynthese um $50 \%$ reduziert, willkürlich gleich eins gesetzt. Abb.3 zeigt die für sämtliche Metalle 
über ein Jahr gemittelte Abhängigkeit der Photosynthesedepression von der relativen Metallkonzentrationserhöhung $C_{\text {rel }}$, wobei

$$
C_{\text {rel }}=\frac{\text { effektive Konzentrationserhöhung }}{C_{24 \mathrm{~h}}^{50 \%}}=\frac{C_{\text {eff }}}{C_{24 \mathrm{~h}}^{C_{0} \%}} .
$$

Bei bekannter $C_{24 \mathrm{~h}}^{50 \%}$ lässt sich mit Hilfe dieser normalisierten, für die beiden Seen praktisch identischen Kurven die toxische Wirkung jeder Konzentrationserhöhung der untersuchten Metalle mit guter Präzision voraussagen (vgl. Abb.4). Wird die Konzentration mehrerer Metalle gleichzeitig erhöht, so ist bei rein additiver Wirkung der einzelnen Metalle $i, k, l, \ldots$

$$
C_{\mathrm{rel}}(\text { tot })=\frac{C_{\mathrm{eff}}(i)}{C_{24 \mathrm{~h}}^{50 \%}(i)}+\frac{C_{\mathrm{eff}}(k)}{C_{24 \mathrm{~h}}^{50 \%}(k)}+\frac{C_{\mathrm{eff}}(l)}{C_{24 \mathrm{~h}}^{50 \%}(l)}+\cdots
$$

und die zu erwartende Photosynthesehemmung lässt sich mit Hilfe der normalisierten Beziehung zwischen der Metallkonzentration und der Photosynthesedepression (Abb. 3) ermitteln. Aus Abb. 4 geht deutlich hervor, dass im Gegensatz zu den Versuchen mit einzelnen Metallen, bei gleichzeitiger Konzentrationserhöhung von $\mathrm{Hg}, \mathrm{Cu}, \mathrm{Cd}, \mathrm{Zn}, \mathrm{Pb}$, die derart abgeschätzten Photosyntheseraten die effektiv gemessenen mehrheitlich überschätzen. Daraus muss abgeleitet werden, dass Metalle in Kombination mit anderen Metallen toxischer wirken als dann, wenn sie nur allein in erhöhter Konzentration auftreten. Dies steht in Übereinstimmung mit den Beobachtungen an höheren Wasserpflanzen von HUTCHINSON und Czyrska [17] und denjenigen von Stockes et al. [32] an Scenedesmus, die ergaben, dass Kupfer die Nickel- und Nickel die Kupferaufnahme förderte.
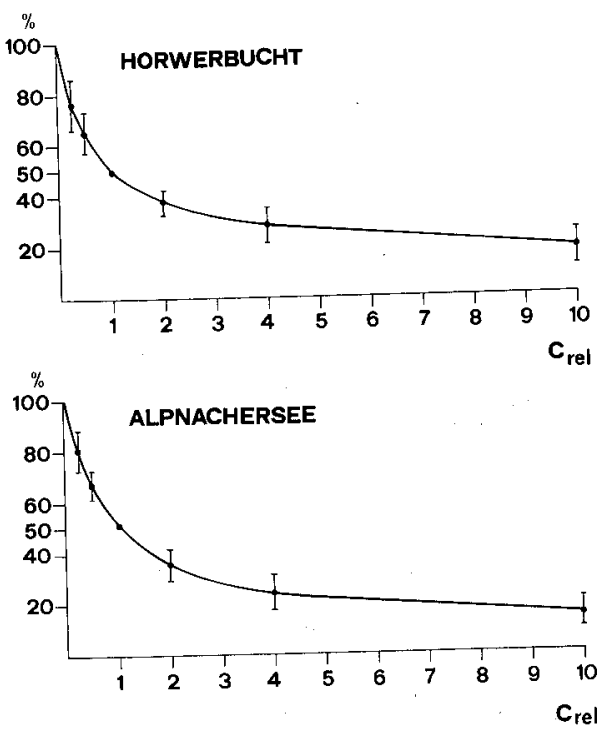

Abb.3. Normalisierte Darstellung der Photosynthesedepression durch Konzentrationserhöhungen von $\mathrm{Hg}, \mathrm{Cu}, \mathrm{Cd}, \mathrm{Zn}, \mathrm{Pb}$.

$C_{\text {rel }}=\frac{\text { effektive Konzentrationserhöhung }}{C_{24 \mathrm{~h}}^{50 \%}}$

Weitere Erklärungen im Text S.00ff.

Fig. 3. Normalized relation between depression of photosynthesis and heavy metal concentration increase

$C_{\text {rel }}=\frac{\text { increase of metal concentration }}{C_{24 \mathrm{~h}}^{50 \%}}$

For further explanation see text p.00ff. and summary. 

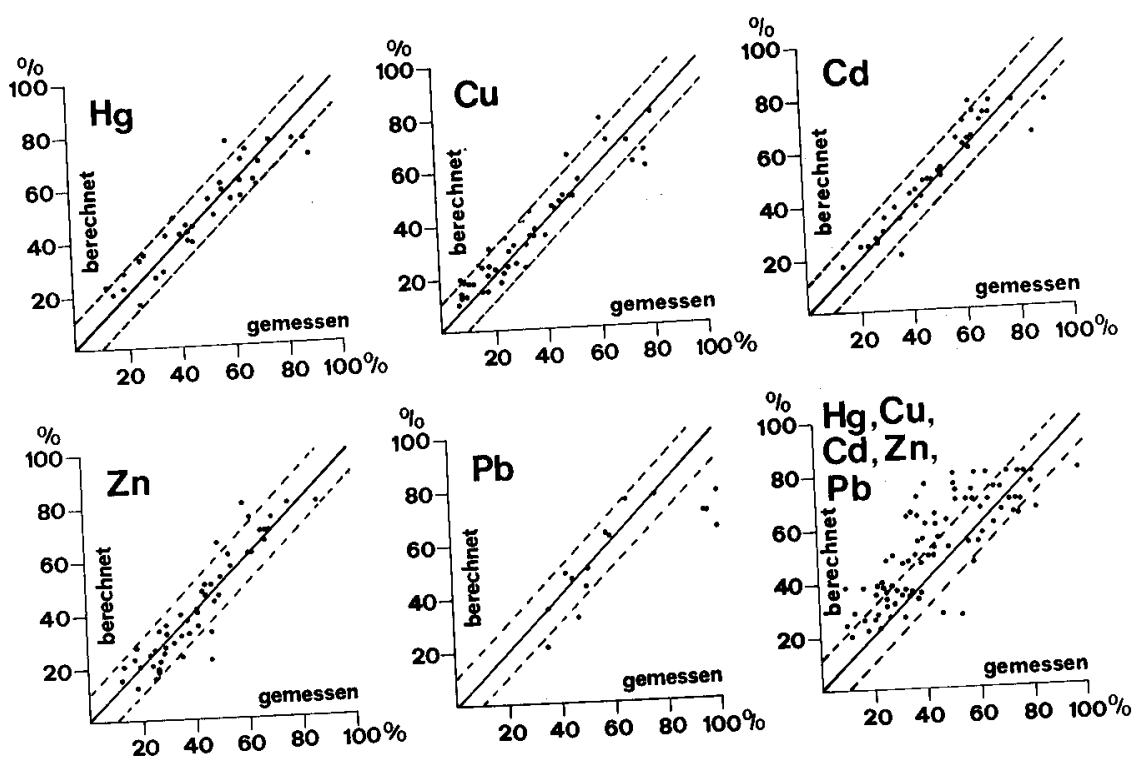

Abb.4. Vergleich von gemessenen und mit Hilfe von Abb. 3 berechneten Photosyntheseraten. Comparison of measured and estimated relative rates of photosynthesis (Control without added metals Comparison of measured and estimated relative rates of photosynthes
equals $100 \%$ ). For concentration increases different from $\mathrm{C}_{24 \mathrm{~h}}^{50 \%}$ (for definition see summary) relative
rates of photosynthesis have been estimated with the help of the relation shown in fig. 3 . When the conall metals were increased simultaneously then $\mathrm{C}_{\text {rel }}$ (total) was assumed to be

$\mathrm{C}_{\mathrm{rel}}($ total $)=\mathrm{C}_{\mathrm{rel}}(\mathrm{Hg})+\mathrm{C}_{\mathrm{rel}}(\mathrm{Cu})+\mathrm{C}_{\mathrm{rel}}(\mathrm{Cd})+\mathrm{C}_{\mathrm{rel}}(\mathrm{Zn})+\mathrm{C}_{\mathrm{rel}}(\mathrm{Pb})$

4.2 Faktoren, welche die toxische Wirkung der Metalle beeinflussen könnten

Die toxische Wirkung der Metalle kann durch die folgenden saisonal variablen Grössen beeinflusst werden:

- qualitative Zusammensetzung des Phytoplanktons

- Phytoplanktonbiomasse

- Konzentration an allochthonen Trübstoffen

- pH-Wert

- Gehalt des Wassers an gelösten oder kolloidalen organischen Komplexbildnern

- Kalziumkonzentration

- Temperatur

4.21 Qualitative Zusammensetzung des Phytoplanktons

Die Photosyntheseleistung $P_{\text {tot }}$ des Gesamtplanktons mit der Biomasse $B_{\text {tot }}$ setzt sich zusammen aus den individuellen Leistungen der einzelnen Arten 1 bis $n$

$$
P_{\text {tot }}=K_{1} \cdot B_{1}+K_{2} B_{2}+K_{3} B_{3}+\cdots K_{n} \cdot B_{n},
$$

wobei $K_{1}$ bis $K_{n}$ artspezifische Konstanten sind. 
Wird die Photosyntheseleistung durch einen Metallstress reduziert, so ergibt sich

$$
P_{\text {tot }}(\mathrm{red})=K_{1} \cdot r_{1} \cdot B_{1}+K_{2} \cdot r_{2} \cdot B_{2}+K_{3} \cdot r_{3} \cdot B_{3}+\cdots K_{n} \cdot r_{n} \cdot B_{n},
$$

wobei die Grösse $r_{n}$ angibt, wie eine bestimmte Konzentrationserhöhung eines Metalls die Photosyntheseleistung der Art $n$ beeinflusst. $K_{n}$ und $r_{n}$ sind zwar artspezifische Konstanten, aber sie sind nicht von der Zeit unabhängig. $K_{n}$ ist abhängig von der Nährstoffkonzentration, der Temperatur sowie vom physiologischen Zustand der Population, und $r_{n}$ wird durch alle oben aufgeführten, saisonal variablen Grössen beeinflusst. Setzt sich die Phytoplanktonbiomasse zum Zeitpunkt $t$ aus $n$ Arten zusammen, so sind zur Bestimmung aller $K$ - und $r$-Werte $2 n$ Gleichungen nötig. Da $n$ stets grösser als 1 ist, bei der gewählten Versuchsanordnung aber immer nur 2 Grössen, nämlich $P_{\text {tot }}$ und $P_{\text {tot }}$ (red) bestimmt wurden, ist es auch bei bekannter Zusammensetzung des Planktons nicht möglich, Rückschlüsse auf die Empfindlichkeit einzelner Planktonarten zu ziehen.

\subsection{Phytoplanktonbiomasse}

Die Beobachtung, dass die toxische Wirkung einer bestimmten Metallkonzentration von der Organismendichte beeinflusst wird [28-30, 37], kann mit der Annahme, dass zwischen den Algen und dem Metall in der Lösung als erster Schritt eine Komplexreaktion

$$
M+A \rightleftarrows M A
$$

stattfinde, plausibel erklärt werden.

Es gilt dann

$$
\begin{aligned}
& {[M]_{\mathrm{tot}}=[M]+[M A]} \\
& {[A]_{\mathrm{tot}}=[A]+[M A]} \\
& \frac{[M A]}{[M][A]}=K,
\end{aligned}
$$

wobei $[M]_{\text {tot }}$ die Gesamtmetallkonzentration und $[A]_{\text {tot }}$ die Ligandkonzentration an der Zelloberfläche bedeutet. $[M]$ steht für jene Metallfraktion, die nicht mit $A$ assoziiert ist, $[A]$ gibt die Konzentration der Liganden an, die nicht mit dem Metall $M$ komplexiert sind, und $K$ ist eine konditionelle Komplexbildungskonstante.

$A_{\text {tot }}$ nimmt proportional zu mit der Zellzahl, und $[M A] /[A]$ ist ein Mass für die pro Zelle aufgenommene Kupfermenge. Der Zusammenhang zwischen diesen beiden Grössen wurde in Abb.5 für einen angenommenen Wert von $K=10^{3}$ dargestellt. Es zeigt sich, dass die pro Zelle aufgenommene Metallmenge unterhalb einer bestimmten Organismendichte von dieser unabhängig ist und bei wachsender Zellzahl anfänglich langsam und schliesslich umgekehrt proportional mit der Zellzahl abnimmt. Es ist daher zu erwarten, dass auch die toxische Wirkung einer bestimmten Metallkonzentration unterhalb einer gewissen Algendichte von der Zellzahl unabhängig ist und bei weiterem Anwachsen der Zelldichte kontinuierlich kleiner wird. 


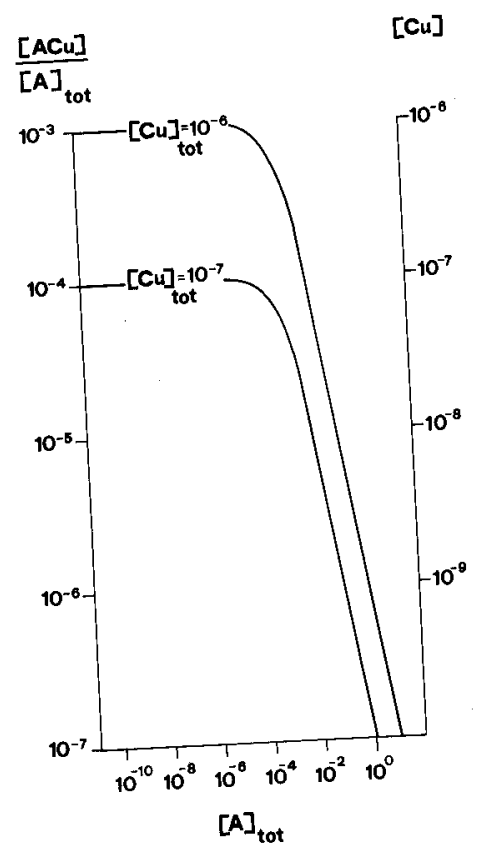

Abb.5. Gleichgewicht zwischen an der Zelloberfläche komplexiertem und im Nährmedium gelöstem Kupfer in Abhängigkeit von der Algenkonzentration (prople Komplexder Gesamtkupferkonzentration. Kildungskonstante $=10^{3}$.

Fig.5. Hypothetical equilibrium concentrations of copper complexed at the surface of algae $\left([A \mathrm{Cu}] /\left[A_{\text {tot }}\right]\right)$ and dissolved in the nutrient medium $([\mathrm{Cu}])$ as a function of $[A]_{\text {tot }}$ and $[\mathrm{Cu}]_{\text {tot }}$, assuming a conditional complex formation constant of $10^{3}$. $[A]_{\text {tot }}$ : total ligand concentration at the cell surface (proportional to cell density). Note that up to a certain algal density, copper uptake and therefore copper toxicity is independent of algal density.

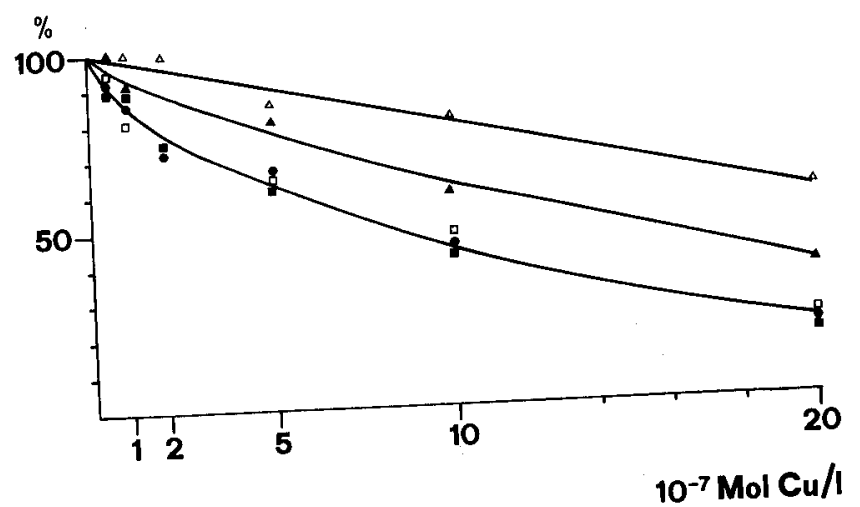

Abb.6. Photosynthesedepression durch Kupfersulfat in Abhängigkeit von der Phytoplanktondichte.

- Unverändertes Seewasser, $[\mathrm{PN}]=35 \mu \mathrm{g} \mathrm{N} / 1$

Mit Phytoplankton angereichertes Seewasser, $[\mathrm{PN}]=400 \mu \mathrm{g} \mathrm{N} / 1$

Mit Phytoplankton angereichertes Seewasser, $[\mathrm{PN}]=900 \mu \mathrm{g}$ N/l

Mit Phytoplankton angereichertes Seewasser, $[\mathrm{PN}]=1740 \mu \mathrm{g} \mathrm{N} / 1$

$\triangle$ Mit Phytoplankton angereichertes Seewasser, $[\mathrm{PN}]=3200 \mu \mathrm{g} \mathrm{N} / 1$

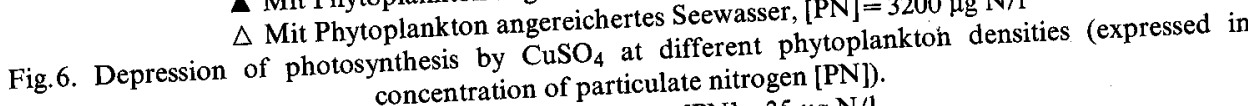

- Unaltered lake water, $[\mathrm{PN}]=35 \mu \mathrm{g} \mathrm{N} / 1$

Lake water increased in phytoplankton density, $[\mathrm{PN}]=400 \mu \mathrm{g} \mathrm{N} / 1$

$\square$ Lake water increased in phytoplankton density, $[\mathrm{PN}]=900 \mu \mathrm{g} \mathrm{N} / 1$

$\Delta$ Lake water increased in phytoplankton density, $[\mathrm{PN}]=1740 \mu \mathrm{g} \mathrm{N} / 1$

$\triangle$ Lake water increased in phytoplankton density, $[\mathrm{PN}]=3200 \mu \mathrm{g} \mathrm{N} / 1$

(Note that under natural conditions [PN] concentration never exceeded $400 \mu \mathrm{g} \mathrm{N} / 1$ ) 
Um zu überprüfen, ob die saisonal variable Phytoplanktondichte für die saisonal veränderliche Metalltoxizität verantwortlich sein könnte, wurde Seewasser mit natürlichem Phytoplankton (gefangen mit einem Phytoplanktonnetz, Maschenweite $50 \mu \mathrm{m})$ angereichert und in diesen Proben die toxische Wirkung von Kupfer in Abhängigkeit von der Algendichte untersucht. Als Mass für die Phytoplanktondichte wurde die Konzentration an partikulärem Stickstoff (PN) bestimmt. Sie betrug im unveränderten Seewasser $35 \mu \mathrm{g} / 1$, in den mit Phytoplankton angereicherten Proben $400 \mu \mathrm{g} / 1,900 \mu \mathrm{g} / 1,1740 \mu \mathrm{g} / 1,3200 \mu \mathrm{g} / \mathrm{l}$. Die Resultate dieses Experimentes (siehe Abb.6) bestätigen die Voraussage des Modells (siehe Abb.5), wonach unterhalb einer bestimmten Planktondichte die Kupferaufnahme pro Zelle und damit die toxische Wirkung des Kupfers von der Zelldichte unabhängig ist.
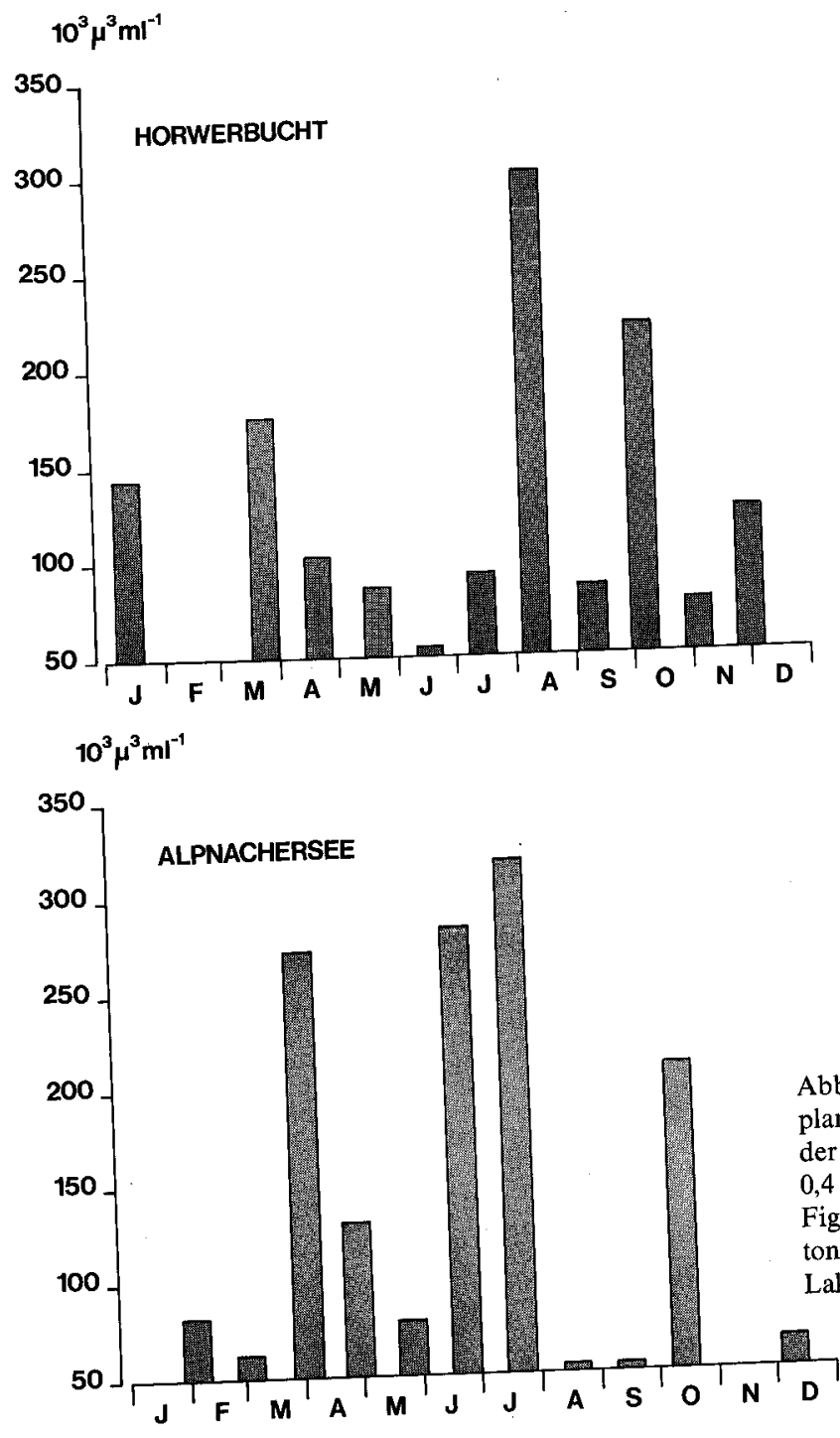
Eine Reduktion der Phytotoxizität wurde erst bei Konzentrationen von mehr als $900 \mu \mathrm{g} / 1$ beobachtet; Konzentrationen also, die im Verlaufe des Jahres weder im Alpnachersee noch in der Horwer Bucht je erreicht wurden [1]. Daraus folgt, dass die saisonal variable toxische Wirkung der Metalle nicht primär mit der saisonal variablen Phytoplanktondichte erklärt werden kann. Ein Vergleich von saisonal variablen Phytoplanktondichte
Abb. 1 und Abb. 7 bestätigt, dass zwischen der stehenden Ernte des Phytoplanktons
und der toxischen Wirkung der Metalle kein direkter Zusammenhang besteht.

\subsection{Allochthone Trübstoffe}

Wenn allochthone Trübstoffe Metalle adsorbieren, so können sie die Verfügbarkeit der Metalle für planktische Organismen beeinflussen. Aufgrund dieser Konkurrenzsituation wäre bei maximaler Konzentration an alloch Nonen Trübstoffen eine minimal toxische Wirkung der Metalle zu erwarten. Nach KRUMMENACHER [20] und BACCINI [1] ist das partikuläre Eisen ein guter lneror für die im See suspendierten allochthonen Trübstoffe. Aus Abb. 8 geh feine Trübstoffeine aus Sedimenten des Zuflusses Grosse Schliere gewensubstanz/Liter oder $420 \mu \mathrm{g}$ fraktion bei einer Konzentratioxische Wirkung des Kupfers nicht zu beeinflussen partikulärem Eisen/Liter Alpnachersee noch in der Horwer Bucht je derart hohe Trübstoffkonzentrationen festgestellt wurden, muss angenommen werden, dass die in den See eingeschwemmten Trübstoffe die Konzentration der gelösten, für das Plankton verfügbaren Metallspezies nicht wesentlich zu beeinflussen vermögen. Plankton verfügbaren Metallspezies nicht wesentien überein, die ergaben, dass in der
Dies stimmt auch mit den Feldbeobachtungen [1]
trophogenen Zone im Verlauf des Jahres die Konzentration an gelösten Metallen trotz erheblichen Konzentrationsschwankungen an Trübstoffen praktisch konstant blieb.

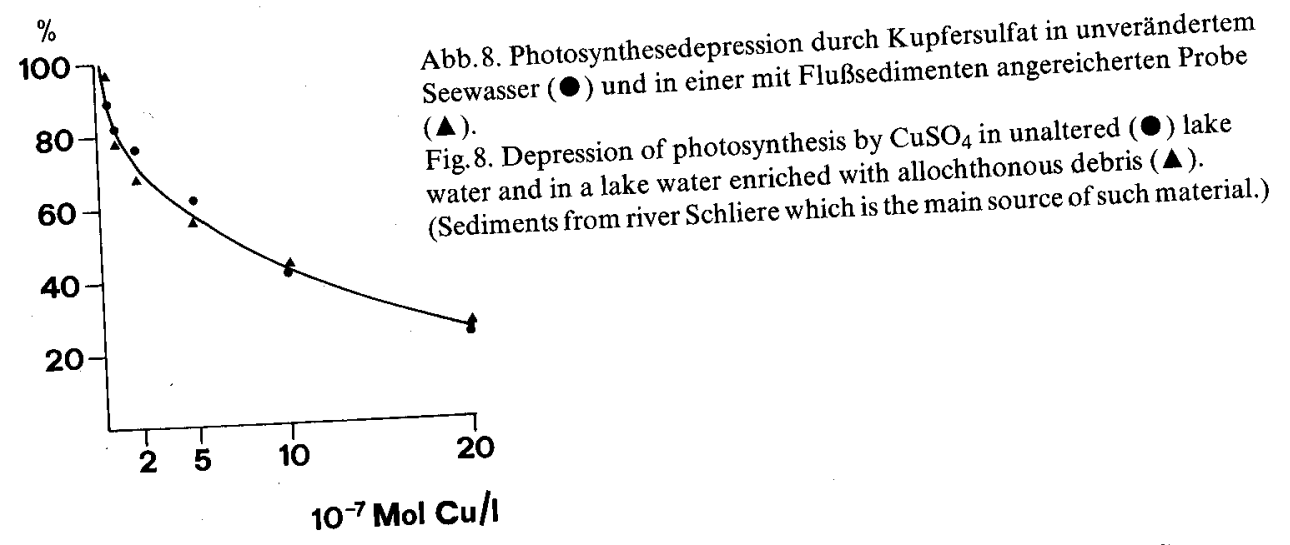

Maximale Konzentrationen an partikulärem Eisen wurden in beiden Seen zur Zeit heftiger Niederschläge in den Zeitabschnitten Juni/Juli und August/September beobachtet [1]. Entgegen der oben aufgestellten Hypothese hemmten aber $\mathrm{Hg}, \mathrm{Cu}$ $\mathrm{Cd}$ und $\mathrm{Zn}$ die planktische Photosynthese in beiden Seen gerade im Juni/Juli 
besonders stark. Umgekehrt wirkten die vier Metalle im März/April bei minimalen Konzentrationen an partikulärem Eisen am wenigsten toxisch. Diese Beobachtungen bestätigen daher den experimentellen Befund, dass die saisonal variable toxische Wirkung der Metalle nicht mit der variablen Konzentration an allochthonen Trübstoffen erklärt werden kann.

\section{$4.24 \mathrm{pH}-$ Wert}

Steemann Nielsen et al. [30] fanden bei Untersuchungen mit Chlorella, dass Kupfer im sauren Nährmedium $(\mathrm{pH}=5)$ deutlich weniger toxisch wirkt als in einem leicht basischen Nährmedium bei $\mathrm{pH} 8$. WUHRMANN und ZoBRIst [37] beobachteten eine zunehmende bakterizide Wirkung von Silber mit ansteigendem $\mathrm{pH}-$ Wert (Bereich pH 6,3 bis 8,7). Eigene Untersuchungen über den Einfluss des pH-Wertes auf die Phytotoxizität des Kupfers, bei denen der $\mathrm{pH}$-Wert durch Begasen des Seewassers mit verschiedenen Luft- $\mathrm{CO}_{2}$-Gemischen variiert wurde, zeigten dagegen oberhalb des $\mathrm{pH}$-Wertes 7,4 eine Abnahme der Toxizität mit ansteigendem $\mathrm{pH}$ Wert (siehe Abb. 9).
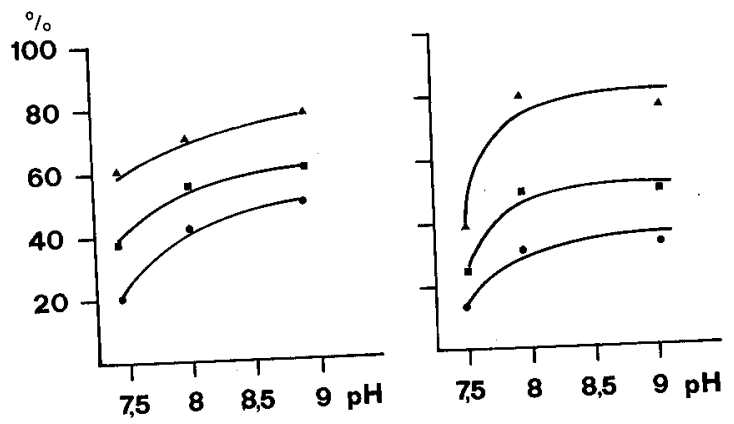

Abb.9. Relative Photosyntheseraten (Kontrolle, ohne Kupferzugabe $=100 \%$ ) in Abhängigkeit von der Kupferkonzentration $\left(\Delta 2 \cdot 10^{-7} \mathrm{Mol} / 1 ; \square \cdot 10^{-7} \mathrm{Mol} / \mathrm{l}\right.$ und $10^{-6} \mathrm{Mol} / \mathrm{l}$ ) und vom $\mathrm{pH}-$ Wert. Fig.9. Rate of photosynthesis as a function of $\mathrm{pH}$ at different copper concentrations

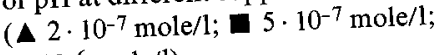
- $10^{-6}$ mole $/ 1$ ).

Die pH-Werte der beiden Seen lagen im Januar/Februar bei 7,6, vom März bis Oktober zwischen 8,1 und 8,7 und im November/Dezember bei etwa 8. Im Alpnachersee fielen zwar die beiden Toxizitätsminima von Kupfer zeitlich zusammen mit hohen $\mathrm{pH}-W e r t e n$ (8,5 Ende April und 8,3 bis 8,5 im Juli/August), gleichzeitig wurde aber Ende Juni für $\mathrm{Hg}, \mathrm{Cu}, \mathrm{Cd}$ und $\mathrm{Zn}$ eine maximal toxische Wirkung beim höchsten $\mathrm{pH}-$ Wert ( $\mathrm{pH} 8,7$ ) beobachtet. Auch in der Horwer Bucht hemmten diese vier Metalle während des Sommers bei maximalen $\mathrm{pH}$-Werten die planktische Photosynthese am stärksten.

Aufgrund der Beobachtung, dass zwar im kontrollierten Experiment die toxische Wirkung des Kupfers im pH-Bereich von 7,5 bis 9 mit zunehmendem $\mathrm{pH}$ abnahm, im Verlaufe eines Jahres aber die Primärproduktion durch sämtliche Metalle während des Sommers bei maximalen $\mathrm{pH}$-Werten am stärksten gehemmt wurde, muss geschlossen werden, dass weder die Protonenkonzentration noch die Totalkonzentration an anorganischem Kohlenstoff ausschlaggebend für die saisonale Variation der Phytotoxizität der Metalle verantwortlich ist. 


\subsection{Organische Liganden}

Da organische Liganden wie EDTA, NTA, aber auch Zitronensäure, Histidin, Glycin oder ein Zooplanktonextrakt die Verfügbarkeit und die Toxizität von Schwermetallen gegenüber dem Phytoplankton herabzusetzen vermögen $[3,7,9$, $25,30]$, ist die Vermutung naheliegend, dass auch ein Teil der im Seewasser vorhandenen organischen Substanzen als Komplexbildner die toxische Wirkung von Metallen beeinflussen könnte.

Im Alpnachersee wurden Ende April ein erstes und im Juli/August ein zweites Konzentrationsmaximum an gelösten organischen Stickstoffverbindungen (GON) beobachtet. In Übereinstimmung damit wurde in diesen Zeitabschnitten die Photosynthese des Planktons durch eine Erhöhung der Kupfer- und Quecksilberkonzentration am wenigsten stark gehemmt. In der Horwer Bucht erreichten die gelösten organischen Stickstoffverbindungen mit $300 \mu \mathrm{g}$ GON/ En bestimmte Konzentratrationsmaximum. Zu diesem Zeitpunkt hemmte aber eine bestich stärker als im Vortionserhöhung eines sich bei Berücksichtigung aller Wertepaare kein gesicherter Zusammenhang zwischen der GON-Konzentration des Seewassers und der photosynthesehemmenden Wirkung des Kupfers ergibt.

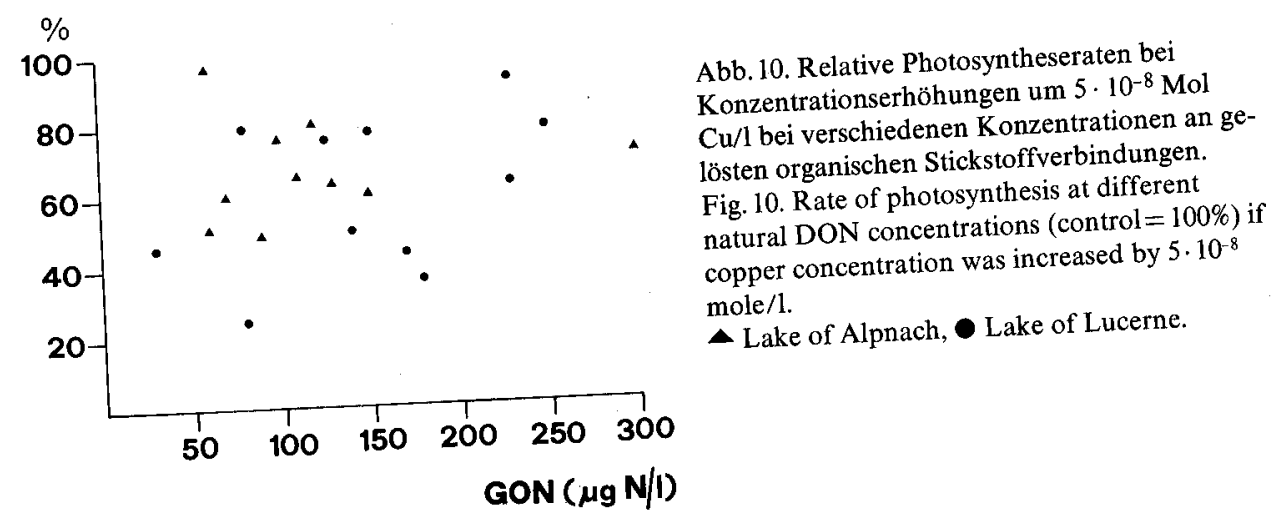

\subsection{Kalziumkonzentration}

Kalzium tritt gegenüber dem Kupfer in etwa 100000mal höherer Konzentration auf. Es ist denkbar, dass es als divalentes Kation bei diesem enormen Konzentrationsüberschuss die Sorption des Kupfers durch das Phytoplankton zu beeinflussen vermag. Die Kalziumkonzentration in der trophogenen Zone des Alpnachersees schwankt zwischen 3,4 mval/1 während der Vollzirkulation und 3,0 mval/1 am Ende der Stagnationsperiode. In der Horwer Bucht liegen die entsprechenden Werte bei 1,9 und 1,7 mval/1. Trotz den deutlichen Unterschieden in der Kalziumhärte der 1,9 und $1,7 \mathrm{mval} / 1$. Trotz den deutlichen Unterschee nicht weniger toxisch als im
beiden Seen wirkte das Kupfer im Alpnachersen
weicheren Wasser der Horwer Bucht (siehe Tabelle 6 ). Anfang März wurde im Alpnachersee die Photosyntheserate des Phytoplanktons durch $10^{-7} \mathrm{Mol} \mathrm{Cu} / 1$ bei einer 
Kalziumkonzentration von 3,4 mval/1 um $80 \%$ reduziert. Ende April bewirkte bei unveränderter Kalziumkonzentration selbst eine $20 \mathrm{mal}$ höhere Kupferkonzentration keine derart starke Reduktion der Photosynthese.

Die experimentelle Untersuchung über die Beeinflussung der Phytotoxizität des Kupfers durch die Ca-Konzentration (siehe Abb.11) ergab in Ubereinstimmung mit den erwähnten Feldbeobachtungen, dass zwischen der während der Sommerstagnation leicht erniedrigten Kalziumkonzentration und der erhöhten toxischen Wirkung des Kupfers sehr wahrscheinlich kein direkter kausaler Zusammenhang besteht.

Tabelle 6. Jahresmittel der relativen Photosyntheseraten (Kontrolle $=100 \%$ ) in Abhängigkeit von der Kupferkonzentration im Alpnachersee und in der Horwer Bucht.

Table 6. Relative rates of photosynthesis (control $=100 \%$ ) depending on copper concentration increase (yearly average).

\begin{tabular}{|c|c|c|c|c|c|c|}
\hline $\begin{array}{l}\text { Konzentrationserhöhung } \\
\text { Increase concentration } \\
\left(10^{-7} \mathrm{Mol} \mathrm{Cu} / 1\right)\end{array}$ & 0.5 & 1 & 2 & 5 & 10 & 20 \\
\hline Alpnachersee (Lake of Alpnach) & $\begin{array}{l}59 \\
66\end{array}$ & $\begin{array}{l}42 \\
51\end{array}$ & $\begin{array}{l}32 \\
38\end{array}$ & $\begin{array}{l}17 \\
23\end{array}$ & $\begin{array}{l}13 \\
15\end{array}$ & $\begin{array}{l}10 \\
10\end{array}$ \\
\hline
\end{tabular}

Horwer Bucht (Lake of Lucerne)

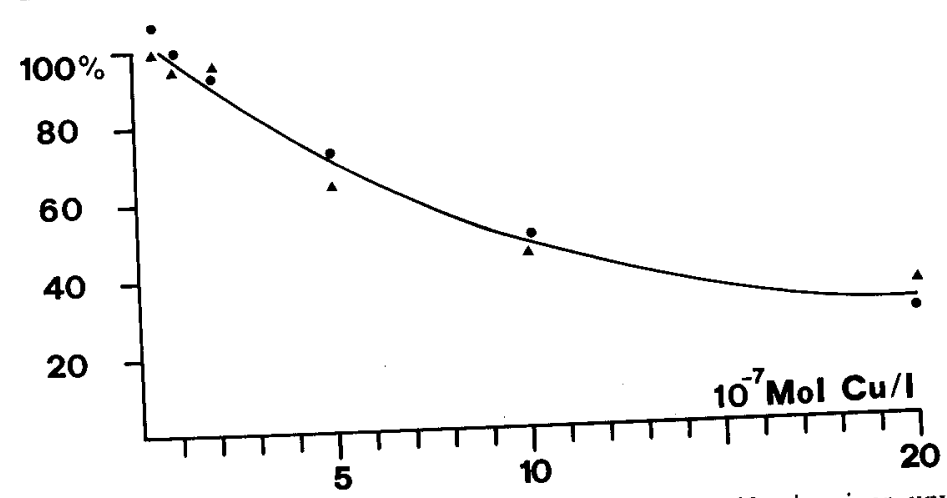

Abb.11. Reduktion der Photosynthese durch Kupfersulfat in einer unveränderten Seewasserprobe (๑, $[\mathrm{Ca}]=1,94 \mathrm{mval} / 1, \mathrm{pH}=7,9)$ und einer mit Kalziumnitrat angereicherten Probe $(\boldsymbol{\Lambda},[\mathrm{Ca}]=$

Fig. 11. Depression of photosynthesis as a function of increased copper concentration in unaltered $(\bullet,[\mathrm{Ca}]=1.94 \mathrm{mval} / 1, \mathrm{pH} 7.9)$ and in calcium enriched samples $(\Delta,[\mathrm{Ca}]=2.9 \mathrm{mval} / \mathrm{l}, \mathrm{pH}=7.8)$.

\subsection{Temperatur}

Aufgrund von Untersuchungen von Mandelli [22] wäre zu erwarten, dass die Sorption von Kupfer durch das Phytoplankton und damit möglicherweise auch die toxische Wirkung dieses Metalls mit ansteigender Temperatur zunehmen würde. Da im Alpnachersee aber sowohl im Winter (Februar und Dezember) als auch im Frühsommer Toxizitätsmaxima auftraten und anderseits Toxizitätsminima sowohl im Frühjahr als auch im Hochsommer beobachtet wurden, kann auch der jahreszeitliche Temperaturgang nicht leztlich für die variable Phytotoxizität der verschiedenen Metalle verantwortlich sein. 


\section{Zusammenfassung und Schlussfolgerungen}

Im Alpnachersee und in der Horwer Bucht wurde die $\mathrm{Hemmung}$ der planktischen Photosynthese durch die anorganischen Metallsalze $\mathrm{HgCl}_{2}, \mathrm{CuSO}, \mathrm{Cd}\left(\mathrm{NO}_{3}\right)_{2}$, $\mathrm{ZnCl}_{2}$ und $\mathrm{Pb}\left(\mathrm{NO}_{3}\right)_{2}$ in monatlichen Abständen während eines Jä einzelnen

- Das Phytoplankton reagierte auf Konzentrationserhom die Photosynthese des talle sehr empfindlich. Quecksilber und Kupfer hem Zink. Blei hemmte im talle sehr empfindlich. Quecksilber und Kupfer hirk toxischer als Zink. Blei hemmte im
Planktons am stärksten. Cadmium wirkte
Vergleich zu den anderen Metallen die Photosynthese des Planktons am schwächsten. - In der trophogenen Zone der beiden Seen schwankte die Metallkonzentration im
Jahresverlauf in folgenden Bereichen:

1 bis $2 \cdot 10^{-8} \mathrm{Mol} \mathrm{Cu} / 1,1$ bis $4 \cdot 10^{-10} \mathrm{Mol} \mathrm{Cd} / 1,2$ bis $6 \cdot 10^{-8} \mathrm{Mol} \mathrm{Zn} / 1$ und 2 bis $6 \cdot 10^{-9} \mathrm{Mol} \mathrm{Pb} / 1$.

- Konzentrationserhöhungen, die während des ganzen Jahres mit grosser Wahrscheinlichkeit gerade noch keinen negativen Einfluss auf das Phytoplankton ausübten, lagen bei $1 \cdot 10^{-9} \mathrm{Mol} \mathrm{Hg} / 1,5 \cdot 10^{-9} \mathrm{Mol} \mathrm{Cu} / 1,2 \cdot 10^{-8} \mathrm{Mol} \mathrm{Cd} / 1,5 \cdot 10^{-8}$

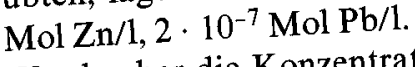

- Wurde aber die Konzentration aller Metalle gleichzeitig erhöht, so wirkten Konzentrationserhöhungen um nur $5 \cdot 10^{-10} \mathrm{Mol} \mathrm{Hg} / 1,5 \cdot 10^{-9} \mathrm{Mol} \mathrm{Cu} / 1,5 \cdot 10^{-9} \mathrm{Mol}$ $\mathrm{Cd} / 1,5 \cdot 10^{-8} \mathrm{Mol} \mathrm{Zn} / 1$ und $5 \cdot 10^{-8} \mathrm{Mol} \mathrm{Pb} / 1$ während des Grossteils des Jahres in beiden Seen toxisch gegenüber dem Phytoplankton. in beiden Seen toxisch gegenüber dem Phytoplankton.
- Bei gleichzeitiger Konzentrationserhöhung aller Metalle verstärkten sich verschie-
dene Metalle in ihrer toxischen Wirkung gegenseitig.

- Ein gesetzlich erlaubter Konzentrationsanstieg in den Zuflüssen um etwa $5 \cdot 10^{-9}$ $\mathrm{Mol} \mathrm{Hg} / 1,1,5 \cdot 10^{-7} \mathrm{Mol} \mathrm{Cu} / 1,4 \cdot 10^{-8} \mathrm{Mol} \mathrm{Cd} / 1,3 \cdot 10^{-6} \mathrm{Mol} \mathrm{Zn/l}$ und 2,4 $10^{-7}$ $\mathrm{Mol} \mathrm{Pb} / 1$ würde in den beiden Seen mit Sicherheit zu Konzentrationen führen, die auf das aktuell vorhandene Plankton im Kurzzeitversuch bereits toxisch wirkten. Um die gesamtökologische Bedeutung erhöhter Metallbelastungen besser abschätzen zu können, wird geplant, diese Kurzzeitexperimente durch Freilandversuche von längerer Dauer zu ergänzen.

- Es wurde experimentell nachgewiesen, dass die natürlicherweise auftretenden Schwankungen der Biomassekonzentration, der Kalziumkonzentration und der Konzentration an allochthonen Trübstoffen die Phytotoxizität der Metalle in den beiden Seen nicht wirksam zu beeinflussen vermögen.

- Eine $\mathrm{pH}$-Steigerung über den $\mathrm{pH}$-Wert von 7,4 hinaus verminderte im kontrollierten Experiment die Phytotoxizität des Kupfers.

- Ein direkter Zusammenhang zwischen der saisonal unterschiedlich toxischen Wirkung der Metalle und den Variablen pH-Wert, Temperatur und Konzentration an gelösten organischen Stickstoffverbindungen konnte nicht nachgewiesen werden.

- Aufgrund der in beiden Seen auffallend synchronen Veränderungen der Empfindlichkeit des Planktons gegenüber Cadmium und Zink einerseits und gegenüber Quecksilber und Kupfer anderseits wird vermutet, dass die Photosynthesehemmung durch Cadmium und Zink und durch Kupfer und Quecksilber auf zwei verschiedenen Wirkungsmechanismen beruhe. 
- Es wird vermutet, dass die Veränderung in der qualitativen Zusammensetzung des Planktons die wichtigste Ursache für die saisonal variable toxische Wirkung der einzelnen Metalle sei und dass umgekehrt eine erhöhte Metallbelastung primär zu einer Verschiebung in der qualitativen Zusammensetzung des Planktons führen würde.

\section{SUMMARY}

Heavy metals are known to be toxic to organisms.

It was the goal of this paper

- to determine the concentrations which had no adverse effects on the natural phytoplankton of two prealpine Swiss lakes (Lake of Alpnach and Lake of Lucerne),

- and to study the influence of several factors such as phytoplankton composition and density, concentration of dissolved calcium and allochthonous debris, $\mathrm{pH}$-value and $\mathrm{DON}$ concentration on heavy metal toxicity.

Over monthly intervals subsurface samples were collected in the two lakes and subdivided into 40 subsamples. In each, the heavy metal concentration was increased as indicated in Table 3 . In the subsamples numbered 36 to 40 , in which the concentration of all the metals was increased simultaneously, the concentration of each metal was ten times lower than in the single metal experiments. Depression of photosynthesis was measured with the $\mathrm{C}_{14}$ method in a light thermostat at the actual lake temperatures.

Figure 1 indicates that the toxic effect of the metals varied seasonally and was somewhat different in the two lakes. A synergistic effect of the different metals is also suggested when their concentrations were increased simultaneously.

The generallized order of metal toxicity towards natural phytoplankton (see Fig. 2) was

$$
\mathrm{Hg} \geqslant \mathrm{Cu}>\mathrm{Cd}>\mathrm{Zn}>\mathrm{Pb} \text {. }
$$

In the single metal experiments the different metals did not adversely affect phytoplankton photosynthesis if the concentration increase above background was equal or below $10^{-9} \mathrm{~mole} \mathrm{Hg} / 1 ; 5 \cdot 10^{-9}$ mole $\mathrm{Cu} / 1 ; 2 \cdot 10^{-8}$ mole $\mathrm{Cd} / \mathrm{l} ; 5 \cdot 10^{-8}$ mole $\mathrm{Zn} / 1$ and $2 \cdot 10^{-7}$ mole $\mathrm{Pb} / 1$. (For indigenous concentrations see Table 2.)

If the concentration of all the metals was increased simultaneously, then $5 \cdot 10^{-10}$ mole $\mathrm{Hg} / 1+5 \cdot 10^{-9}$ mole $\mathrm{Cu} / \mathrm{l}+5 \cdot 10^{-9}$ mole $\mathrm{Cd} / \mathrm{l}+5 \cdot 10^{-8}$ mole $\mathrm{Zn} / 1+5 \cdot 10^{-8}$ mole $\mathrm{Pb} / \mathrm{l}$ reduced photosynthesis significantly most often (see Table 5).

Setting the increase in concentration $C_{50 \%}^{24 h}$ which reduced photosynthesis by $50 \%$ equal to 1 , it was possible to normalize the relation between metal concentration and inhibition of photosynthesis (see Fig. 3) for all the metals. When depression of photosynthesis was estimated with the help of these curves and the known $C_{50 \%}^{24 h}$, very good agreement with the experimental results was found for the single metal experiments (see Fig.4). When the concentrations of all the metals were increased simultaneously, then the depression of photosynthesis was generally underestimated by this method. It is thus deduced that inhibition of photosynthesis was not only the result of an additive effect of the toxicity of different metals. Rather, it suggests that some metals have a synergistic effect on the toxicity of the metal mixture.

It was shown experimentally that the natural fluctuations of phytoplankton density, concentration of dissolved calcium and allochthonous debris did not affect heavy metal toxicity (see Fig. 6, 8, 11).

In controlled experiments an increase of the $\mathrm{pH}$-value above 7.4 decreased the inhibitory effect of copper towards photosynthesis (see Fig.9), but it was impossible to relate the seasonal variation of heavy metal toxicity either to $\mathrm{pH}$, to temperature or to DON concentration.

It is believed that seasonal changes in phytoplankton composition are the main reasons for seasonal variations in the toxic effect of heavy metals, and that an increased metal loading would primarily change the phytoplankton composition. 


\section{RESUME}

Des échantillons de surface ont été prélevés mensuellement dans les lacs d'Alpnach et des QuatreCantons (baie de Horw).

Dans ces échantillons, on a augmenté la concentration métallique tel qu'indique le tableau 3 et on a examiné l'effet de cette augmentation sur la photosynthèse planctonique. La figure 1 montre que l'effet toxique des métaux variait saisonnièrement et que les métaux lourds réagissent synergétiquement lors d'une augmentation simultanée de la concentration.

En général, on a constaté la gradation toxique suivante:

$$
\mathrm{Hg} \geqslant \mathrm{Cu}>\mathrm{Cd}>\mathrm{Zn}>\mathrm{Pb} .
$$

Des augmentations de concentration de $10^{-9} \mathrm{~mole} \mathrm{Hg} / 1,5 \cdot 10^{-9} \mathrm{~mole} \mathrm{Cu} / 1,2 \cdot 10^{-8} \mathrm{~mole} \mathrm{Cd} / 1,5 \cdot 10^{-8}$ mole $\mathrm{Zn} / 1$ et $2 \cdot 10^{-7}$ mole $\mathrm{Pb} / 1$ ne montraient jamais d'effet nocif sur le phytoplancton naturel (voir fig.2). D'autre part, la photosynthèse planctonique a été réduite si on augmentait la concentration métallique simultanément de $5 \cdot 10^{-10}$ mole $\mathrm{Hg} / \mathrm{l}+5 \cdot 10^{-9} \mathrm{~mole} \mathrm{Cu} / 1+5 \cdot 10^{-9} \mathrm{~mole} \mathrm{Cd} / 1+5 \cdot 10^{-8} \mathrm{~mole}$ $\mathrm{Zn} / 1+5 \cdot 10^{-8}$ mole $\mathrm{Pb} / 1$ (voir tableau 5). On a montré expérimentalement que les fluctuations naturelles de la densité de plancton, des concentrations de calcium et du débris allochtone n'influencaient pas l'action toxique du cuivre (voir fig.6, 8, 11). Une augmentation du $\mathrm{pH}$ réduisait l'effet toxique du cuivre (fig.9).

On suppose que la composition de phytoplancton changeant saisonnièrement serait la cause principale de la variation saisonnière de l'action toxique des métaux et qu'une augmentation de la charge métallique d'un lac résulterait primairement dans un changement de la composition des espèces phytoplanctoniques.

\section{LITERATURVERZEICHNIS}

[1] BACCINI, P., Untersuchungen über den Schwermetallhaushalt in Seen, Schweiz. Z. Hydrol, 38, 121158 (1976).

[2] BAILS, J.D., Mercury in Fish on the Great Lakes, in: Environmental Mercury Contamination (Eds R. Hartung und R. D. Dinman; Science Publishers Inc.; Ann Arbor 1972), S.31-37.

[3] Barber, R.T., und Ryther, J.H., Organic Chelators: Factors Affecting Primary Production in the Cromwell Current Uppwelling, J. exp. Mar. Biol. Ecol. 3, 191-199 (1969).

[4] Biesinger, K.E., und Christensen, G. M., Effects of various Metals on Survival, Growth, Reproduction and Metabolism of Daphnia magna, J. Fish. Res. Bd., Canada 29, 1991-1700 (1972).

[5] Bowen, H.J.M., Trace Elements in Biochemistry (Academic Press London, New York 1966), 235 S.

[6] BringmanN, G., und KÜHN, R., Vergleichende abwassertoxikologische Untersuchungen an Bakterien, Algen und Kleinlebewesen, Gesundheitsingenieur 80, 115-120 (1959).

[7] BUNDI, TH., Untersuchungen über die Aufnahme verschiedener Kupferspezies durch Algen, Diss. ETH, in Vorbereitung (1976).

[8] Doudoroff, P., und Katz, M., Critical Review of Literature on the Toxicity of Industrial Wastes and Their Components to Fish. II The Metal as Salts, Sew. ind. Wast. 25, 802-839 (1953).

[9] Erickson, S.J., Lackie, N., und MaloneY, T.E., A Screeming Technique for Estimating Copper Toxicity to Estuarine Phytoplankton, J. WPCF 42, R270-R278 (1970)

[10] Erickson, S.J., Maloney, T.E., und Gentile, J.H., Effect of NTA on the Growth and Metabolism of Estuarine Phytoplankton, J. WPCF 42, R329-R335 (1970).

[11] Förstner, U., und MülLER, G., Schwermetalle in Flüssen und Seen als Ausdruck der Umweltverschmutzung (Springer Verlag, Berlin 1974).

[12] Gächter, R., Phosphorhaushalt und planktische Primärproduktion im Vierwaldstättersee (Horwer Bucht) Schweiz. Z. Hydrol. 30, 1-66 (1968).

[13] GIBson, C.E., The Algicidal Effect of Copper on a Green and a Blue-Green Algae and Some Ecological Implications, J. appl. Ecol. 9, 513-518 (1972).

[14] Greig, R.A., und Seagran, H.L., Survey of Mercury Concentrations in Fishes of Lakes St.Clair, Erie and Huron, in: Environmental Mercury Contamination, (Eds R. Hartung und B.D. Dinman; Science Publishers Inc., Ann Arbor 1972), S.38-45. 
[15] Horne, A.J., und Goldman, Ch.R., Suppression of Nitrogen Fixation by Blue-Green Algae in a Eutrophic Lake with Trace Additions of Copper, Science 183, 409-411 (1974).

[16] Hutchinson, T.C., Comparative Studies of the Toxicity of Heavy Metals to Phytoplankton and Their Synergistic Interactions, Wat. Pollut. Res., Canada 8, 68-90 (1973).

[17] Hutchinson, T.C., und Czyrska, H., Heavy Metal Toxicity and Synergism to Floating Aquatic Weeds, Verh. int. Ver. Limnol. 19, 2102-2111 (1975).

[18] Hynes, H.B.N., The Biology of Polluted Waters (Liverpool University Press 1960).

[19] Kobayashi, J., Relation between the "Itai-Itai" Disease and the Pollution of River Water by Cadmium from a Mine, Advances in Water Pollution Research, Proc. 5th Intern. Conf., San Francisco and Haway 1970, Vol. 1, I 25, 1-7 (1971).

[20] Krummenacher, T., Die Nährstoffbilanz des Alpnachersees, Diss. ETH (1976).

[21] Manahan, S.E., und Smith, M.J., Copper Micronutrient Requirement for Algae, Envir. Sci. Technol. 7, 829-833 (1973).

[22] Mandelli, E.F., The Inhibitory Effects of Copper on Marine Phyto-Plankton, Marine Sci. 14, 47-57 (1969).

[23] Pagenkopf, G.H., Russo, R.C., und Thurston, R.V., Effect of Complexation on the Toxicity of Copper to Fishes, J. Fish. Res. Bd., Canada 31, 462-465 (1974).

[24] RiLey, J.P., und Roth, I., The Distribution of Trace Elements in Some Species of Phytoplankton, J. mar. biol. Ass. U.K. 51, 63-72 (1971).

[25] Soeder, D.J., Schulze, G., und ThIele, D., Einfluss verschiedener Kulturbedingungen auf das Wachstum in Synchronkulturen von Chlorella fusca, Str. Kr. Arch. Hydrobiol. Suppl. 33, 127-171 (1967).

[26] Sunda, W., The Relationship between Cupric Ion Activity and the Toxicity of Copper to Phytoplankton, Diss. (Massachusetts Institute of Technology, 1975).

[27] Stadelmann, P., Stickstoffkreislauf und Primärproduktion im mesotrophen Vierwaldstättersee (Horwer Bucht) und im eutrophen Rotsee, mit besonderer Berücksichtigung des Nitrats als limitierendem Faktor, Schweiz. Z. Hydrol. 33, 1-65 (1971).

[28] Steemann Nielsen, E., und Wium-Andersen, S., Copper Ions as Poison in the Sea and Freshwater, Marine Biol. 6, 93-97 (1970).

[29] Steemann Nielsen, E., Kamp-Nielsen, L., und Wium-Andersen, S., The Effect of Deleterious Concentrations of Copper on the Photosynthesis of Chlorella pyrenoidosa, Physiol. Plant. 22, 1121 bis 1133 (1969).

[30] Steemann Nielsen, E., und Kamp-Nielsen, L., Influenca of Deleterious Concentrations of Copper on the Growth of Chlorella pyrenoidosa, Physiol. Plant. 23, 828-840 (1970).

[31] Steemann Nielsen, E., und Wium-Andersen, S., The Influenca of $\mathrm{Cu}$ on Photosynthesis and Growth in Diatoms, Physiol. Plant. 24, 480-484 (1971).

[32] STokes, P.M., Hutchinson, T.C., und KRAUTER, K., Heavy Metal Tolerance in Algae Isolated from Polluted Lakes Near the Sudbury, Ontario smelters, Wat. Pollut., Canada 8, 178-201 (1973).

[33] TAKeUCHI, T., Distribution of Mercury in the Environment of Minamata Bay and the Inland Ariaka Sea, in: Environmental Mercury Contamination (Eds R. Hartung und B.D. Dinman; Science Publishers Inc., Ann Arbor 1972), S. 79-81.

[34] Eidg. Departement des Innern: Verordnung über Abwassereinleitungen vom 8. Dezember 1975.

[35] Winner, R.W., VAN Dike, J., CARIS, N., und FARReL, M.P., Response of Macroinvertebrate Fauna to a Copper Gradient in a Experimentally-Polluted Stream, Verh. Int. Ver. Limnol. 19, 2121-2127 (1975).

[36] Wuhrmann, K., und Eichenberger, E., Experiments on the Effects of Inorganic Enrichment of Rivers on Periphyton Primary Production. Verh. int. Ver. Limnol. 19, 2028-2034 (1975).

[37] WuhrmanN, K., und ZoBrist, F., Untersuchung über die bakterizide Wirkung von Silber im Wasser, Schweiz. Z. Hydrol. 20, 218-254 (1958).

Adresse des Autors:

Dr. R. Gächter, Seenforschungslaboratorium der EAWAG/ETH, CH-6047 Kastanienbaum. 